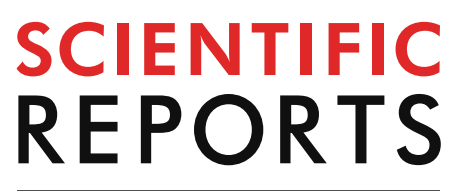

natureresearch

Check for updates

\title{
GC-MS analysis
}

of phytoconstituents

from Amomum nilgiricum

and molecular docking interactions of bioactive serverogenin acetate with target proteins

\author{
Narasimhamurthy Konappa ${ }^{1,5}$, Arakere C. Udayashankar ${ }^{1,5}$, Soumya Krishnamurthy ${ }^{2}$, \\ Chamanalli Kyathegowda Pradeep ${ }^{1}$, Srinivas Chowdappa ${ }^{3}$ \& Sudisha Jogaiah ${ }^{4}{ }^{4}$
}

Amomum nilgiricum is one of the plant species reported from Western Ghats of India, belonging to the family Zingiberaceae, with ethno-botanical values, and is well-known for their ethno medicinal applications. In the present investigation, ethyl acetate and methanol extracts of $A$. nilgiricum were analyzed by Fourier transform infrared spectrometer (FTIR) and gas chromatography-mass spectrometry (GC-MS) to identify the important functional groups and phytochemical constituents. The FTIR spectra revealed the occurrence of functional characteristic peaks of aromatic amines, carboxylic acids, ketones, phenols and alkyl halides group from leaf and rhizome extracts. The GC-MS analysis of ethyl acetate and methanol extracts from leaves, and methanol extract from rhizomes of A. nilgiricum detected the presence of 25 phytochemical compounds. Further, the leaf and rhizome extracts of $A$. nilgiricum showed remarkable antibacterial and antifungal activities at $100 \mathrm{mg} / \mathrm{mL}$. The results of DPPH and ferric reducing antioxidant power assay recorded maximum antioxidant activity in $A$. nilgiricum methanolic leaf extract. While, ethyl acetate leaf extract exhibited maximum $\alpha$-amylase inhibition activity, followed by methanolic leaf extract exhibiting aldose reductase inhibition. Subsequently, these 25 identified compounds were analyzed for their bioactivity through in silico molecular docking studies. Results revealed that among the phytochemical compounds identified, serverogenin acetate might have maximum antibacterial, antifungal, antiviral, antioxidant and antidiabetic properties followed by 2,4-dimethyl-1,3-dioxane and $\left(1,3-{ }^{13} \mathrm{C}_{2}\right)$ propanedioic acid. To our best knowledge, this is the first description on the phytochemical constituents of the leaves and rhizomes of $A$. nilgiricum, which show pharmacological significance, as there has been no literature available yet on GC-MS and phytochemical studies of this plant species. The in silico molecular docking of serverogenin acetate was also performed to confirm its broad spectrum activities based on the binding interactions with the antibacterial, antifungal, antiviral, antioxidant and antidiabetic target proteins. The results of the present study will create a way for the invention of herbal medicines for several ailments by using $A$. nilgiricum plants, which may lead to the development of novel drugs.

\footnotetext{
${ }^{1}$ Department of Studies in Biotechnology, University of Mysore, 570 006, Manasagangotri, Mysuru, Karnataka, India. ${ }^{2}$ Department of Microbiology, Field Marshal K. M. Cariappa College, A Constituent College of Mangalore University, Madikeri 571201, Karnataka, India. ${ }^{3}$ Department of Microbiology and Biotechnology, Jnanabharathi Campus, Bangalore University, Bengaluru 560 056, Karnataka, India. ${ }^{4}$ Laboratory of Plant Healthcare and Diagnostics, PG Department of Biotechnology and Microbiology, Karnataka University, Dharwad, Karnataka, India. ${ }^{5}$ These authors contributed equally: Narasimhamurthy Konappa and Arakere C. Udayashankar ${ }^{\square}$ email: jsudish@kud.ac.in
} 
Modern-day synthetic and chemical drugs are often explored with hesitate as they exhibit side effects ${ }^{1}$, while traditional herbals are gaining huge interests as they are more natural, environment-friendly and devoid of side effects $^{2}$. Hence, with all the benefits of modern synthetic medicines, people have still preferred plant-based natural medicines over synthetic medicines ${ }^{3}$. Most of the medicinal plants are distinctive in their ability to treat, as well as to cure various human ailments owing to the contribution of various valuable phytoconstituents present in different plant parts ${ }^{3-5}$. In India, from ancient time, different parts of medicinal plants $(\sim 80,000$ species $)$ have been used as traditional medicines in different systems of Indian medicines for treatments of various diseases ${ }^{6}$. At present, about $25 \%$ of the active constituents have been identified from medicinal plants, which have been used as prescribed medicines ${ }^{7}$. Certain reports have estimated that over 25,000 of actual plant-based formulations are available in the Indian systems of folk and traditional medicine, which are prescribed by about 1.5 million practitioners in preventive, persuasive and healing applications ${ }^{7,8}$.

Various bioactive compounds of medicinal plants exhibit stimulating pharmacological actions like antibacterial, antifungal, anticancer, anti-inflammatory and antioxidant properties ${ }^{3,49}$. The potential of these bioactive compounds should be analyzed for their candidature in treatments of various ailments ${ }^{4,7}$. Plant-based medicines are often prepared from crude plant extracts comprising of complex mixture of different phytochemicals. These phytochemicals have unique and complex structures, and are used in treating prolonged as well as contagious diseases $^{2,7}$. An enormous pool of bioactive secondary metabolites exists in various plant species, but merely a small proportion of them have been examined and sustained to be significant source of bioactive agents. In the search for new compounds, and also for quality control, development of suitable screening methods is very important ${ }^{10}$. Extractions and characterizations of numerous such bioactive compounds from various medicinal plants have led to the delivery of certain medicines with high-activity profile ${ }^{11}$.

The initial screening of medicinal plants by spectrometric and chromatographic methods provides basic information on chemical and pharmacological activities, which helps to select the biologically active plants ${ }^{12}$. In recent years, Fourier-transform infrared (FTIR) and gas chromatography-mass spectrometry (GC-MS) has commonly been employed for detection of functional groups and identification of various bioactive therapeutic compounds that are present in medicinal plants ${ }^{13,14}$. GC-MS is one of the best, fast and accurate techniques to detect various compounds, including alcohols, alkaloids, nitro compounds, long chain hydrocarbons, organic acids, steroids, esters and amino acids ${ }^{15}$, and requires a small volume of plant extracts. Hence, in the present study, GC-MS technique was adopted for detection and identification of phytochemical compounds present in the medicinal plant, Amomum nilgiricum belonging to Zingiberaceae family. A. nilgiricum is a recent plant species found in the Western Ghats of Kerala, India ${ }^{16}$. Zingiberaceae is generally known as a family of spicy plants, and numerous members of this family have been used in Ayurvedic and other natural systems of medicine in India ${ }^{17}$. This family includes 53 genera and 1200 species $^{18}$ that are known as ornamentals in different parts of the world, and have been broadly used as medicinal, traditional, food and ornamental plants in many regions of Asia, of which about 250 species are represented in India ${ }^{16,18}$.

Many drugs have failed to enter market owing to their poor pharmacokinetic properties, which incurs huge losses to pharmaceutical companies ${ }^{19}$. Computer-aided tools have emerged as advanced methods for drug discovery, which can be applied to screen the drugs from phytochemicals found in various medicinal plants ${ }^{20}$. Computational prediction models, also known as predictive tools, which play an important part in the selection of methodologies directing pharmaceutical and technological research, and have also been used in in silico prediction of pharmacological, pharmacokinetic and toxicological performance ${ }^{21}$. Currently, molecular docking is an effective and inexpensive method for designing and testing the drugs. This technique provides information about drug receptor interactions that are useful to predict the binding orientation of drug candidates to their target proteins ${ }^{22}$. Besides, this approach helps in systemic study by introducing a molecule on the binding spot of the object macromolecule in a non-covalent fashion, leading to an accurate binding at the active sites of each ligand ${ }^{23}$.

Therefore, the present study focuses on identification of bioactive compounds from ethyl acetate and methanol extracts from A. nilgiricum leaves, and methanol extract from A. nilgiricum rhizomes by GC-MS analysis. We have also evaluated the efficacy of leaves and rhizome extracts of $A$. nilgiricum for their antimicrobial, antioxidant and antidiabetic assay. Subsequently, in silico molecular docking and computational molecular simulation was explored for analysis of the potential bioactive compounds for their antibacterial, antifungal, antiviral, antioxidant and antidiabetic (e.g. $\alpha$-glucosidase, $\alpha$-amylase and aldose reductase) activities. Findings suggest that out of 25 identified phytochemical compounds, serverogenin acetate ([3,12-diacetyloxy-10,14-dimethyl-13-oxo15-(5-oxo-2H-furan-3-yl)-2-oxapentacyclo[9.7.0.01,3.05,10.014,18] octadecan-7-yl] acetate) might have the best pharmacological properties. This report helps predict the structure and formula of phytochemical components present in various solvent extracts of $A$. nilgiricum plants.

\section{Results}

Preliminary phytochemical screening. The phytochemical study of extracts from Amomum nilgiricum leaves and rhizomes revealed a broad variety of phytochemicals. The key phytochemical components, such as flavonoids, anthocyanins, tannins, carbohydrates, alkaloids, cardiac glycosides, steroids, phenols, anthroquinone, leucoanthocyanin, diterpenes and saponins, were present in both the ethyl acetate and methanol extracts (Table 1).

Fourier transform infrared spectrometer (FTIR). The results of FTIR spectra confirmed the presence of functional groups in methanolic leaf extract of A. nilgiricum with peaks at $3421.22 \mathrm{~cm}^{-1}$ (alcohols, phenols), $2924.71 \mathrm{~cm}^{-1}$ (alkanes), $1630.91 \mathrm{~cm}^{-1}$ (amines), $1546.54 \mathrm{~cm}^{-1}$ (alkanes), $1309.39 \mathrm{~cm}^{-1}$ (alcohols, carboxylic acids, esters, ethers), $1383.61 \mathrm{~cm}^{-1}$ (alkanes), $1075.05 \mathrm{~cm}^{-1}$ (aliphatic amines), $890.81 \mathrm{~cm}^{-1}$ (aromatics) and $530.23 \mathrm{~cm}^{-1}$ (alkyl halides) (Fig. 1a). The peaks at $3854.58 \mathrm{~cm}^{-1}, 3843.87 \mathrm{~cm}^{-1}, 3807.71 \mathrm{~cm}^{-1}$ (non-bonded, alcohols, phe- 


\begin{tabular}{|l|l|l|l|}
\hline \multirow{2}{*}{ Phytochemicals } & \multicolumn{2}{|l|}{ Leaf extract } & Rhizome extract \\
\cline { 2 - 4 } & Methanol & Ethyl acetate & Methanol \\
\hline Carbohydrates & + & + & + \\
\hline Flavonoids & + & + & + \\
\hline Cardiac glycosides & + & + & + \\
\hline Anthocyanin & - & - & + \\
\hline Tannins & + & + & + \\
\hline Alkaloids & + & + & + \\
\hline Saponins & - & - & + \\
\hline Steroids & + & + & + \\
\hline Terpenoids & - & + & - \\
\hline Phenols & + & + & + \\
\hline Leucoanthocyanin & + & + & + \\
\hline Proteins and amino acids & + & + & - \\
\hline Diterpenes & + & + & + \\
\hline Anthroquinone & - & - & + \\
\hline
\end{tabular}

Table 1. Phytochemical prescreening of Amomum nilgiricum rhizome and leaf extracts. The marks '+' and '-' indicate 'present' and 'absent', respectively.

nols), $3771.92 \mathrm{~cm}^{-1}$ (carboxylic acids), $3430.65 \mathrm{~cm}^{-1}$ (alcohols, phenols), $2878.32 \mathrm{~cm}^{-1}$ (alkanes), $1653.64 \mathrm{~cm}^{-1}$ (alkenes), $1599.36 \mathrm{~cm}^{-1}$ (aromatics), $1420.58 \mathrm{~cm}^{-1}$ (aromatics), $1380.53 \mathrm{~cm}^{-1}$ (alkanes), $1321.95 \mathrm{~cm}^{-1}$ (alcohols, carboxylic acids, esters, ethers), $1261.17 \mathrm{~cm}^{-1}$ (aromatic amines), $1154.94 \mathrm{~cm}^{-1}$ (alkyl halides), $1076.61 \mathrm{~cm}^{-1}$ (aliphatic amines), $1030.83 \mathrm{~cm}^{-1}$ (aliphatic amines) and $608.52 \mathrm{~cm}^{-1}$ (alkyl halides) determined the existence of functional groups ethyl acetate leaf extract (Fig. 1b).

Likewise, the FTIR spectrum from A. nilgiricum methanolic rhizome extract showed the presence of functional groups with peaks at $3421.17 \mathrm{~cm}^{-1}$ (alcohols, phenols), $2926.01 \mathrm{~cm}^{-1}$ (alkanes), $1634.07 \mathrm{~cm}^{-1}$ (amines), $1553.35 \mathrm{~cm}^{-1}$ (nitro compounds), $1409.21 \mathrm{~cm}^{-1}$ (aromatics), $1089.19 \mathrm{~cm}^{-1}$ (aliphatic amines), $893.20 \mathrm{~cm}^{-1}$ (aromatics), $537.82 \mathrm{~cm}^{-1}$ (alkyl halides) (Fig. 1c).

Gas chromatography-mass spectrometry (GC-MS) analysis. The GC-MS chromatogram of methanol and ethyl acetate leaf extracts of A. nilgiricum recorded a total of 15 peaks corresponding to the bioactive compounds that were recognized by relating their peak retention time, peak area (\%), height (\%) and mass spectral fragmentation patterns to that of the known compounds described by the National Institute of Standards and Technology (NIST) library. Results revealed that 9 and 6 compounds were identified in methanol and ethyl acetate $A$. nilgiricum leaf extracts, respectively (Table $2 \& 3$ ). The GC-MS chromatogram of the methanol extract from A. nilgiricum rhizomes recorded 10 peaks (Table 4). Overall, the structure of 25 phytocompounds identified in the ethyl acetate and methanol extracts from leaves, and from the methanol extract from rhizomes of $A$. nilgiricum are presented in Table 5 along with their retention time.

The phytoconstituents in the methanol leaf extract of $A$. nilgiricum were found to be 5 -aminooxypentanoic acid, 3,7,9-trioxatricyclo[4.2.1.0,4]nonane, 1-(2,4,4-trimethylpentan-2-yl)-4-[4-(2,4,4-trimethylpentan-2-yl) phenoxy]benzene, trimethyl-(2-trimethylsilylphenyl)silane, 3,5-bis(trimethylsilyl)cyclohepta-2,4,6-trien-1one, trimethyl-[4-[2-methyl-4-(4-trimethylsilyloxyphenyl)pent-4-en-2-yl]phenoxy]silane, trimethyl-[4-[4(4-trimethylsilyloxyphenyl)hexan-3-yl]phenoxy] silane, trimethyl-[[4-(trimethylsilylmethyl)phenyl]methyl] silane and 1,1,1,3,5,5,5-heptamethyltrisiloxane (Fig. 2a,b, Table 2). The phytoconstituents in ethyl acetate leaf extract of $A$. nilgiricum were octadec-1-yne, 3,4-heptadien-2-one, 3-cyclopentyl-6-methyl-, [3,12-diacetyloxy10,14-dimethyl-13-oxo-15-(5-oxo-2H-furan-3-yl)-2-oxapentacyclo[9.7.0.01,3.05,10.014,18] octadecan-7-yl] acetate, trimethyl-[4-(2,4,4-trimethylpentan-2-yl)phenoxy] silane, trimethyl-(2-trimethylsilylphenyl)silane, and 3,5-bis(trimethylsilyl)cyclohepta-2,4,6-trien-1-one (Fig. 3a,b, Table 3).

The GC-MS chromatogram of methanol extract from A. nilgiricum rhizome recorded 10 peaks corresponding to bioactive compounds, which were recognized by comparing its mass spectra along with their analogs reported in the NIST library (Table 4). The major components present in A. nilgiricum were ethyl 2-oxopropanoate, $\left(1,3-{ }^{13} \mathrm{C}_{2}\right)$ propanedioic acid, 2-butyl-4-[(E)-pentadec-4-enyl]-1,3,2-dioxaborinan-5-amine, 2,4-dimethyl-1,3-dioxane, 5-amino-6-nitroso- $1 H$-pyrimidine-2,4-dione, $(2 R, 4 S, 5 S, 6 R)$-3,8,9-trioxatricyclo[4.2.1.0 $\left.0^{2,4}\right]$ nonan-5-ol, $(2 S, 4 S, 5 R, 6 R)$-3,8,9-trioxatricyclo[4.2.1.0 $\left.0^{2,4}\right]$ nonan-5-ol, 5-methylhex-3-yn-2-ol and trimethylsilyl tetracosanoate (Fig. 4a,b, Table 4).

Antibacterial activity. Antibacterial activities of leaf and rhizome extracts of A. nilgiricum were tested against different bacterial pathogens at different concentrations $(25,50$ and $100 \mathrm{mg} / \mathrm{mL})$. Maximum zone of inhibition of $21.43 \mathrm{~mm}$ was recorded with ethyl acetate leaf extract against Pseudomonas aeruginosa and highest zone of inhibition was observed for methanolic rhizome extract against Ralstonia solanacearum with $20.72 \mathrm{~mm}$ at $100 \mathrm{mg} / \mathrm{mL}$ as compared to other tested concentrations (Fig. 5). 

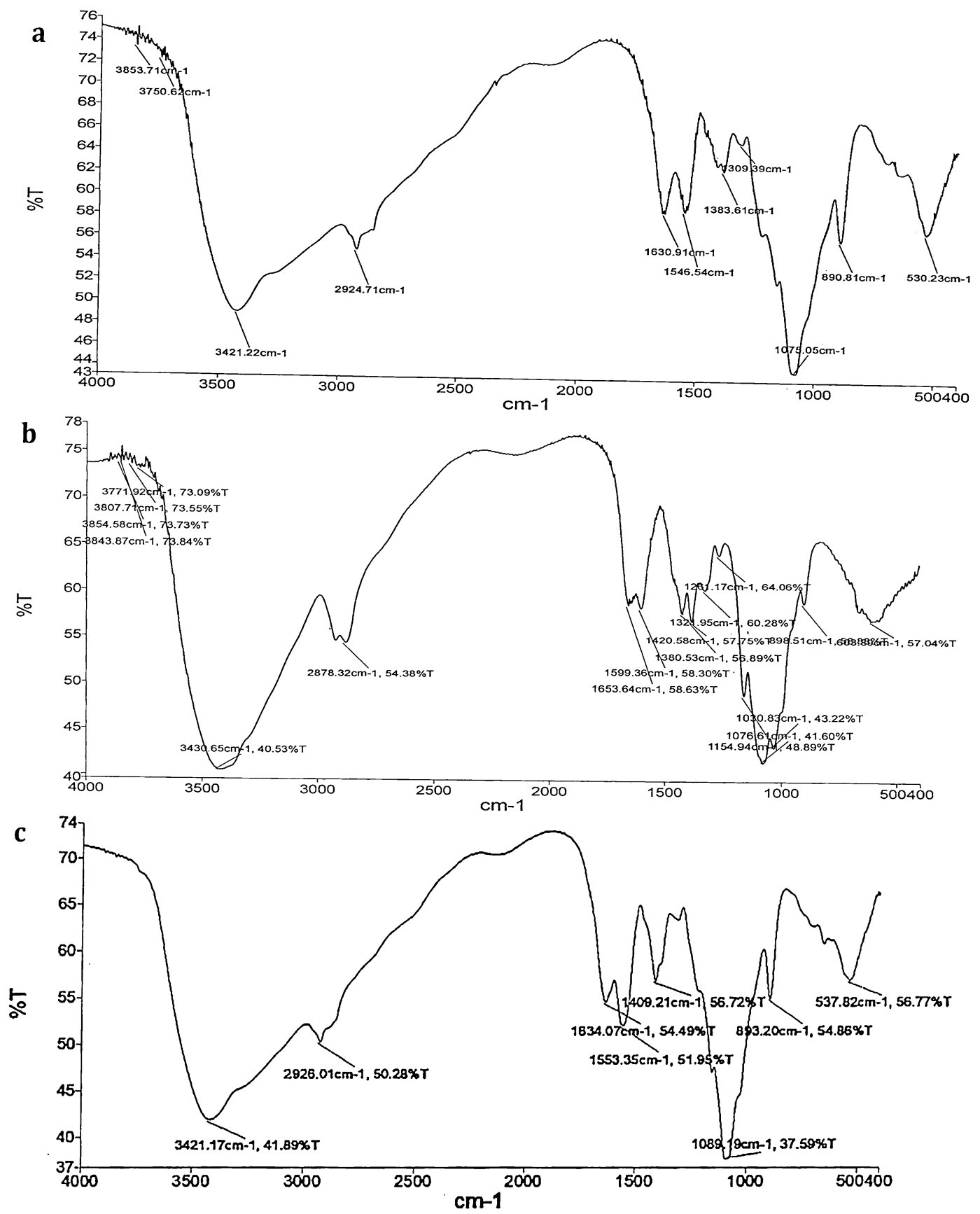

Figure 1. (a) FTIR spectrum from methanolic leaf extract of Amomum nilgiricum. (b) FTIR spectrum from ethyl acetate leaf extract of Amomum nilgiricum. (c) FTIR spectrum from methanolic rhizome extract of Amomum nilgiricum.

Antifungal activity. The ethyl acetate leaf extract of $A$. nilgiricum recorded maximum zone of inhibition of $16.69 \mathrm{~mm}$ against Alternaria alternate. While, methanolic rhizome extract at $100 \mathrm{mg} / \mathrm{mL}$ showed inhibition of $16.32 \mathrm{~mm}$ against Pyricularia oryzae $(16.32 \mathrm{~mm})$ when compared to other tested concentrations used in the study (Fig. 6).

Antioxidant activity. In the present study, the different concentrations of leaf and rhizome extracts of $A$. nilgiricum were subjected to 2,2-diphenyl-1-picryl-hydrazyl-hydrate (DPPH) free radical scavenging method. The methanolic leaf extract was revealed maximum DPPH activity of $83.68 \%$, while gallic acid, ethyl acetate leaf and methanolic rhizome extracts recorded about $94.85 \%, 81.86 \%$ and $84.53 \%$, respectively (Fig. $7 \mathrm{a}$ ). The methanolic leaf extract exhibited the highest DPPH radical scavenging activity with an $\mathrm{IC}_{50}$ of $50.65 \mu \mathrm{g} / \mathrm{ml}$, followed by methanolic rhizome and ethyl acetate extracts with an $\mathrm{IC}_{50}$ values of $59.42 \mu \mathrm{g} / \mathrm{ml}$ and $78.54 \mu \mathrm{g} / \mathrm{ml}$ respectively, whereas the gallic acid exhibited $\mathrm{IC}_{50}$ values of $48 \mu \mathrm{g} / \mathrm{ml}$. The percent Ferric reducing antioxidant power assay 


\begin{tabular}{|c|c|c|c|c|c|}
\hline S1. No & CAS & Name of the compound & Molecular formula & Molecular weight & Peak area $(\%)$ \\
\hline 1 & $5699-55-8$ & 5-aminooxypentanoic acid & $\mathrm{C}_{5} \mathrm{H}_{11} \mathrm{O}_{3} \mathrm{~N}$ & 133.14 & 4.082 \\
\hline 2 & $40,838-15-1$ & 3,7,9-trioxatricyclo[4.2.1.1.0.4] nonane & $\mathrm{C}_{6} \mathrm{H}_{8} \mathrm{O}_{3}$ & 128.13 & 19.379 \\
\hline 3 & $78,721-87-6$ & $\begin{array}{l}\text { 1-(2,4,4-trimethylpentan-2-yl)-4-[4-(2,4,4-tri- } \\
\text { methylpentan-2-yl)phenoxy]benzene }\end{array}$ & $\mathrm{C}_{17} \mathrm{H}_{30} \mathrm{OSi}$ & 278.50 & 3.129 \\
\hline 4 & $17,151-09-6$ & Trimethyl-(2-trimethylsilylphenyl)silane & $\mathrm{C}_{12} \mathrm{H}_{22} \mathrm{Si}_{2}$ & 222.47 & 3.171 \\
\hline 5 & $900,161-21-8$ & 3,5-bis(trimethylsilyl)cyclohepta-2,4,6-trien-1-one & $\mathrm{C}_{13} \mathrm{H}_{22} \mathrm{OSi}_{2}$ & 250.48 & 4.369 \\
\hline 6 & $900,283-56-8$ & $\begin{array}{l}\text { Trimethyl-[4-[2-methyl-4-(4-trimethylsilyloxyphe- } \\
\text { nyl)pent-4-en-2-yl]phenoxy] silane }\end{array}$ & $\mathrm{C}_{24} \mathrm{H}_{36} \mathrm{O}_{2} \mathrm{Si}_{2}$ & 412.7 & 38.631 \\
\hline 7 & $70,244-15-4$ & $\begin{array}{l}\text { Trimethyl-[4-[4-(4-trimethylsilyloxyphenyl) } \\
\text { hexan-3-yl]phenoxy] silane }\end{array}$ & $\mathrm{C}_{24} \mathrm{H}_{38} \mathrm{O}_{2} \mathrm{Si}_{2}$ & 414.72 & 16.246 \\
\hline 8 & $13,183-70-5$ & $\begin{array}{l}\text { Trimethyl-[[4-(trimethylsilylmethyl)phenyl] } \\
\text { methyl]silane }\end{array}$ & $\mathrm{C}_{14} \mathrm{H}_{26} \mathrm{Si}_{2}$ & 250.47 & 6.405 \\
\hline 9 & $1873-88-7$ & 1,1,1,3,5,5,5-heptamethyltrisiloxane & $\mathrm{C}_{7} \mathrm{H}_{22} \mathrm{O}_{2} \mathrm{Si}_{3}$ & 221.5 & 4.588 \\
\hline
\end{tabular}

Table 2. Phytochemical constituents identified in the methanol leaf extract of Amomum nilgiricum using gas chromatography-mass spectrometry. CAS chemical abstract service, $S l$. No. serial number.

\begin{tabular}{|c|c|c|c|c|c|}
\hline Sl. no & CAS & Name of the compound & Molecular formula & Molecular weight & Peak area $(\%)$ \\
\hline 1 & $629-89-0$ & Octadec-1-yne & $\mathrm{C}_{18} \mathrm{H}_{34}$ & 250.5 & 2.852 \\
\hline 2 & $63,922-50-9$ & 3,4-heptadien-2-one, 3-cyclopentyl-6-methyl- & $\mathrm{C}_{13} \mathrm{H}_{20} \mathrm{O}$ & 192.3 & 3.027 \\
\hline 3 & $900,253-62-2$ & $\begin{array}{l}\text { [3,12-diacetyloxy-10,14-Dimethyl-13-oxo-15-(5-oxo-2H-furan-3-yl)-2-oxapentacy- } \\
\text { clo[9.7.0.01,3.05,10.014,18] octadecan-7-yl] acetate }\end{array}$ & $\mathrm{C}_{29} \mathrm{H}_{36} \mathrm{O}_{10}$ & 544.6 & 82.105 \\
\hline 4 & $78,721-87-6$ & Trimethyl-[4-(2,4,4-trimethylpentan-2-yl)phenoxy]silane & $\mathrm{C}_{17} \mathrm{H}_{30} \mathrm{OSi}$ & 278.51 & 3.757 \\
\hline 5 & $17,151-09-6$ & Trimethyl-(2-trimethylsilylphenyl)silane & $\mathrm{C}_{12} \mathrm{H}_{22} \mathrm{Si}_{2}$ & 222.47 & 4.706 \\
\hline 6 & $900,161-21-8$ & 3,5-bis(trimethylsilyl)cyclohepta-2,4,6-trien-1-one & $\mathrm{C}_{13} \mathrm{H}_{22} \mathrm{OSi}_{2}$ & 250.48 & 3.553 \\
\hline
\end{tabular}

Table 3. Phytochemical constituents identified in the ethyl acetate leaf extract of Amomum nilgiricum using gas chromatography-mass spectrometry. CAS chemical abstract service, Sl. No. serial number.

\begin{tabular}{|l|l|l|l|l|l|}
\hline Sl. no & CAS & Name of the compound & Molecular formula & Molecular weight & Peak area (\%) \\
\hline 1 & $617-35-6$ & Ethyl 2-oxopropanoate & $\mathrm{C}_{5} \mathrm{H}_{8} \mathrm{O}_{3}$ & 116.11 & 2.271 \\
\hline 2 & $141-82-2$ & $\left(1,3-{ }^{13} \mathrm{C}_{2}\right)$ propanedioic acid & $\mathrm{C}_{3} \mathrm{H}_{4} \mathrm{O}_{4}$ & 104.06 & 6.142 \\
\hline 3 & $900,143-55-6$ & $\begin{array}{l}\text { 2-butyl-4-[(E)-pentadec-4-enyl]-1,3,2-dioxa- } \\
\text { borinan-5-amine }\end{array}$ & $\mathrm{C}_{22} \mathrm{H}_{44} \mathrm{O}_{2} \mathrm{NB}$ & 365.4 & 18.518 \\
\hline 4 & $766-20-1$ & 2,4 -dimethyl-1,3-dioxane & $\mathrm{C}_{6} \mathrm{H}_{12} \mathrm{O}_{2}$ & 116.16 & 12.222 \\
\hline 5 & $900,270-67-7$ & 5 -amino-6-nitroso-1H-pyrimidine-2,4-dione & $\mathrm{C}_{4} \mathrm{H}_{4} \mathrm{O}_{3} \mathrm{~N}_{4}$ & 156.1 & 9.325 \\
\hline 6 & $900,129-98-9$ & $\begin{array}{l}(2 R, 4 S, 5 S, 6 R)-3,8,9-\text { trioxatricyclo[4.2.1.0 } \\
\text { nonan-5-ol }\end{array}$ & $\mathrm{C}_{6} \mathrm{H}_{8} \mathrm{O}_{4}$ & 144.12 & 33.323 \\
\hline 7 & $900,129-96-9$ & $\begin{array}{l}(1 R, 2 S, 4 R, 5 R)-3,7,9-\text {-trioxatricyclo[4.2.1.0 } \\
\text { nonan-5-ol }\end{array}$ & $\mathrm{C}_{6} \mathrm{H}_{8} \mathrm{O}_{4}$ & 144.12 & 3.300 \\
\hline 8 & $900,129-98-0$ & $\begin{array}{l}(2 S, 4 S, 5 R, 6 R)-3,8,9-\text { trioxatricyclo[4.2.1.0 } \\
\text { nonan-5-ol }\end{array}$ & $\mathrm{C}_{6} \mathrm{H}_{8} \mathrm{O}_{4}$ & 144.12 & 9.621 \\
\hline 9 & $23,293-50-7$ & 5 -methylhex-3-yn-2-ol & $\mathrm{C}_{7} \mathrm{H}_{12} \mathrm{O}$ & 112.17 & 2.818 \\
\hline 10 & $74,367-37-6$ & Trimethylsilyl tetracosanoate & $\mathrm{C}_{27} \mathrm{H}_{56} \mathrm{O}_{2} \mathrm{Si}$ & 440.8 \\
\hline
\end{tabular}

Table 4. Phytochemical constituents identified in the methanol rhizome extract of Amomum nilgiricum using gas chromatography-mass spectrometry. CAS chemical abstract service, Sl. No. serial number.

(FRAP) activity of leaf and rhizome extracts recorded 18.56 to $81.57 \%$ (Fig. $7 \mathrm{~b}$ ). Among extracts, methanolic leaf extract revealed a maximum FRAP activity with an $\mathrm{IC}_{50}$ value was $60.65 \mu \mathrm{g} / \mathrm{ml}$, ethyl acetate leaf extract was showed $\mathrm{IC}_{50}$ value of $79.79 \mu \mathrm{g} / \mathrm{ml}$ and methanolic rhizome extracts was showed $\mathrm{IC}_{50}$ value of $73.42 \mu \mathrm{g} / \mathrm{ml}$. The $\mathrm{IC}_{50}$ value of the extract was less than that of the standard.

Antidiabetic activity. A dose dependent inhibitory action on $\alpha$-amylase activity was observed from the leaf and rhizome of A. nilgiricum extracts (Fig. 8a). Among the extracts, ethyl acetate extract exhibited maximum $\alpha$ - amylase inhibition with $77.22 \%$ ( $\mathrm{IC}_{50} 76.53 \mu \mathrm{g} / \mathrm{ml}$ ), followed by $68.22 \%\left(\mathrm{IC}_{50} 89.43 \mu \mathrm{g} / \mathrm{ml}\right.$ ) recorded for methanolic leaf extract and methanolic rhizome extract showed $55.12 \%\left(\mathrm{IC}_{50} 99.38 \mu \mathrm{g} / \mathrm{ml}\right)$. The standard Acarbose showed $\alpha$-amylase inhibition of $87.17 \%\left(\mathrm{IC}_{50} 0.32 \mu \mathrm{g} / \mathrm{ml}\right)$. A dose dependent activity of $\alpha$-glucosidase 


\begin{tabular}{|c|c|c|c|}
\hline Sl. no & Retention time & Name of the compound & Structure \\
\hline 1 & RT-20.986 & 5-aminooxypentanoic acid & $\mathrm{C}(\mathrm{CCON}) \mathrm{CC}(=\mathrm{O}) \mathrm{O}$ \\
\hline 2 & RT-21.916 & 3,7,9-trioxatricyclo[4.2.1.1.2,4]nonane & $\mathrm{C}_{1} \mathrm{C}_{2} \mathrm{C}\left(\mathrm{O}_{2}\right) \mathrm{C}_{3} \mathrm{COC}_{1} \mathrm{O}_{3}$ \\
\hline 3 & RT-27.368 & $\begin{array}{l}\text { 1-(2,4,4-trimethylpentan-2-yl)-4-[4-(2,4,4-trimethylpentan-2-yl)phenoxy] } \\
\text { benzene }\end{array}$ & $\mathrm{CC}(\mathrm{C})(\mathrm{C}) \mathrm{CC}(\mathrm{C})(\mathrm{C}) \mathrm{C}_{1} \mathrm{CCC}\left(\mathrm{CC}_{1}\right) \mathrm{O}[\mathrm{Si}](\mathrm{C})(\mathrm{C}) \mathrm{C}$ \\
\hline 4 & RT-27.753 & Trimethyl-(2-trimethylsilylphenyl)silane & $\mathrm{C}[\mathrm{Si}](\mathrm{C})(\mathrm{C}) \mathrm{C}_{1} \mathrm{CCCCC}_{1}[\mathrm{Si}](\mathrm{C})(\mathrm{C}) \mathrm{C}$ \\
\hline 5 & RT-27.898 & 3,5-bis(trimethylsilyl)cyclohepta-2,4,6-trien-1-one & {$\left[\mathrm{SiH}_{3}\right] \mathrm{C}_{1} \mathrm{CC}(=\mathrm{O}) \mathrm{CCC}\left(\left[\mathrm{SiH}_{3}\right]\right) \mathrm{C}_{12}$} \\
\hline 6 & RT-28.043 & $\begin{array}{l}\text { Trimethyl-[4-[2-methyl-4-(4-trimethylsilyloxyphenyl)pent-4-en-2-yl] } \\
\text { phenoxy]silane }\end{array}$ & $\begin{array}{l}\mathrm{CC}(\mathrm{C})\left(\mathrm{CC}(=\mathrm{C}) \mathrm{C} 1=\mathrm{CC}=\mathrm{C}\left(\mathrm{C}=\mathrm{C}_{1}\right) \mathrm{O}[\mathrm{Si}](\mathrm{C})(\mathrm{C}) \mathrm{C}\right) \mathrm{C}_{2}=\mathrm{CC}=\mathrm{C}\left(\mathrm{C}=\mathrm{C}_{2}\right) \mathrm{O}[\mathrm{Si}] \\
(\mathrm{C})(\mathrm{C}) \mathrm{C}\end{array}$ \\
\hline 7 & RT-28.288 & Trimethyl-[4-[4-(4-trimethylsilyloxyphenyl)hexan-3-yl]phenoxy]silane & $\mathrm{CCC}\left(\mathrm{C}_{1} \mathrm{CCC}\left(\mathrm{CC}_{1}\right) \mathrm{O}[\mathrm{Si}](\mathrm{C})(\mathrm{C}) \mathrm{C}\right) \mathrm{C}(\mathrm{CC}) \mathrm{C}_{2} \mathrm{CCC}\left(\mathrm{CC}_{2}\right) \mathrm{O}[\mathrm{Si}](\mathrm{C})(\mathrm{C}) \mathrm{C}_{1}$ \\
\hline 8 & RT-28.489 & Trimethyl-[[4-(trimethylsilylmethyl)phenyl]methyl]silane & {$\left[\mathrm{SiH}_{3}\right] \mathrm{C}_{1} \mathrm{CCC}\left(\left[\mathrm{SiH}_{3}\right]\right) \mathrm{CC}$} \\
\hline 9 & RT-28.589 & 1,1,1,3,5,5,5-heptamethyltrisiloxane & $\mathrm{C}[\mathrm{SIH}](\mathrm{O}[\mathrm{Si}](\mathrm{C})(\mathrm{C}) \mathrm{C}) \mathrm{O}[\mathrm{Si}](\mathrm{C})(\mathrm{C}) \mathrm{C}$ \\
\hline 10 & RT-19.950 & Octadec-1-yne & ССССССССССССССССС \#С \\
\hline 11 & RT-21.086 & 3,4-heptadien-2-one, 3-cyclopentyl-6-methyl- & $\mathrm{CC}(\mathrm{C}) \mathrm{C}=\mathrm{C}=\mathrm{C}(\mathrm{C} 1 \mathrm{CCCC} 1) \mathrm{C}(=\mathrm{O}) \mathrm{C}$ \\
\hline 12 & RT-21.891 & \begin{tabular}{|l} 
[3,12-diacetyloxy-10,14-dimethyl-13-oxo-15-(5-oxo-2H-furan- \\
3-yl)-2-oxapentacyclo[9.7.0.01,3.05,10.014,18] octadecan-7-yl] acetate
\end{tabular} & 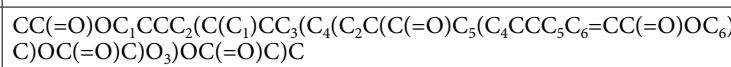 \\
\hline 13 & RT-27.098 & Trimethyl-[4-(2,4,4-trimethylpentan-2-yl)phenoxy]silane & $\mathrm{CC}(\mathrm{C})(\mathrm{C}) \mathrm{CC}(\mathrm{C})(\mathrm{C}) \mathrm{C}_{1} \mathrm{CCC}\left(\mathrm{CC}_{1}\right) \mathrm{O}[\mathrm{Si}](\mathrm{C})(\mathrm{C}) \mathrm{C}$ \\
\hline 14 & RT-27.718 & Trimethyl-(2-trimethylsilylphenyl)silane & $\mathrm{C}[\mathrm{Si}](\mathrm{C})(\mathrm{C}) \mathrm{C}_{1} \mathrm{CCCCC}_{1}[\mathrm{Si}](\mathrm{C})(\mathrm{C}) \mathrm{C}$ \\
\hline 15 & RT-27.783 & 3,5-bis(trimethylsilyl)cyclohepta-2,4,6-trien-1-one & $\mathrm{C}[\mathrm{Si}](\mathrm{C})(\mathrm{C}) \mathrm{C}_{1}=\mathrm{CC}\left(=\mathrm{CC}(=\mathrm{O}) \mathrm{C}=\mathrm{C}_{1}\right)[\mathrm{Si}](\mathrm{C})$ \\
\hline 16 & RT-4.759 & Ethyl 2-oxopropanoate & $\operatorname{CCOC}(=\mathrm{O}) \mathrm{C}(=\mathrm{O}) \mathrm{C}$ \\
\hline 17 & RT-7.880 & $\left(1,3-{ }^{13} \mathrm{C}_{2}\right)$ propanedioic acid & $\mathrm{C}(\mathrm{C}(=\mathrm{O}) \mathrm{O}) \mathrm{C}(=\mathrm{O}) \mathrm{O}$ \\
\hline 18 & RT-15.473 & 2-butyl-4-[(E)-pentadec-4-enyl]-1,3,2-dioxaborinan-5-amine & $\mathrm{B}_{1}\left(\mathrm{OCC}\left(\mathrm{C}\left(\mathrm{O}_{1}\right) \mathrm{CCC} / \mathrm{C}=\mathrm{C} / \mathrm{CCCCCCCCCC}\right) \mathrm{N}\right) \mathrm{CCCC}$ \\
\hline 19 & RT-16.399 & 2,4-dimethyl-1,3-dioxane & $\mathrm{CC}_{1} \mathrm{CCOC}\left(\mathrm{O}_{1}\right) \mathrm{C}$ \\
\hline 20 & RT-16.989 & 5-amino-6-nitroso-1 $\mathrm{H}$-pyrimidine-2,4-dione & $\mathrm{Cl}\left(\mathrm{C}\left([\mathrm{NH}] \mathrm{C}(=\mathrm{O})[\mathrm{NH}] \mathrm{C}_{1}=\mathrm{O}\right) \mathrm{N}=\mathrm{O}\right) \mathrm{N}$ \\
\hline 21 & RT-18.084 & $(2 R, 4 S, 5 S, 6 R)-3,8,9$-trioxatricyclo[4.2.1.0 $\left.0^{2,4}\right]$ nonan-5-ol & $\mathrm{C}_{1} \mathrm{C}_{2} \mathrm{C}\left(\mathrm{C}_{3} \mathrm{C}\left(\mathrm{O}_{3}\right) \mathrm{C}\left(\mathrm{O}_{1}\right) \mathrm{O}_{2}\right) \mathrm{O}$ \\
\hline 22 & RT-18.635 & $(1 R, 2 S, 4 R, 5 R)-3,7,9$-trioxatricyclo[4.2.1.0 $\left.0^{2,4}\right]$ nonan-5-ol & $\mathrm{C}_{1} \mathrm{C}_{2} \mathrm{C}_{3} \mathrm{C}\left(\mathrm{O}_{3}\right) \mathrm{C}\left(\mathrm{C}\left(\mathrm{O}_{1}\right) \mathrm{O}_{2}\right) \mathrm{O}$ \\
\hline 23 & RT-18.755 & $(2 S, 4 S, 5 R, 6 R)-3,8,9$-trioxatricyclo[4.2.1.0 $\left.0^{2,4}\right]$ nonan-5-ol & $\mathrm{C}_{1} \mathrm{C}_{2} \mathrm{C}\left(\mathrm{C} 3 \mathrm{C}\left(\mathrm{O}_{3}\right) \mathrm{C}\left(\mathrm{O}_{1}\right) \mathrm{O}_{2}\right) \mathrm{O}$ \\
\hline 24 & RT-19.430 & 5-methylhex-3-yn-2-ol & $\mathrm{CC}(\mathrm{C}) \mathrm{C} \# \mathrm{CC}(\mathrm{C}) \mathrm{O}$ \\
\hline 25 & RT-21.911 & Trimethylsilyl tetracosanoate & 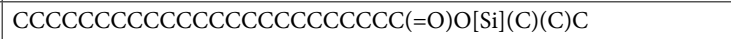 \\
\hline
\end{tabular}

Table 5. Structures and retention time ( $\mathrm{min}$ ) of phytochemical constituents identified in the ethyl acetate and methanol extracts of Amomum nilgiricum leaves, and the methanol extract of A. nilgiricum rhizomes using gas chromatography-mass spectrometry. Sl. No. serial number.

enzyme inhibition was noticed with standard Acarbose (Fig. 8b). The ethyl acetate leaf extract exhibited maximum $\alpha$-glucosidase inhibition was $85.26 \%\left(\mathrm{IC}_{50} 72.54 \mu \mathrm{g} / \mathrm{ml}\right)$, followed by the methanol leaf extract with $78.34 \%$ $\left(\mathrm{IC}_{50} 87.32 \mu \mathrm{g} / \mathrm{ml}\right)$ and methanolic rhizome extract $71.66 \%\left(\mathrm{IC}_{50} 99.46 \mu \mathrm{g} / \mathrm{ml}\right)$. While, standard Acarbose showed a-glucosidase inhibition of $89.54 \%$ ( $\mathrm{IC}_{50} 0.46 \mu \mathrm{g}$ ). Among extracts, the methanolic leaf extract showed highest 87.22\%inhibition of aldose reductaseas compared to other extracts (Fig. 8c). In case of ethyl acetate leaf extract and methanolic rhizome extracts, a inhibition of 81.31 and $73.66 \%$ of aldose reductase was documented in the present study. The aldose reductase inhibition with an $\mathrm{IC}_{50}$ value from ethyl acetate, methanol leaf and methanolic rhizome extracts revealed 77.54, 48.7 and $99.32 \mu \mathrm{g} / \mathrm{ml}$ respectively. While, Quercetin recorded best activity of $7.95 \mu \mathrm{g} / \mathrm{ml}$ with $\mathrm{IC}_{50}$ value.

In silico studies. The GC-MS analysis revealed that A. nilgiricum leaf and rhizome extracts contained 25 bioactive compounds (Table 5). These phytocompounds were analyzed for activities against bacterial, fungal, viral, antioxidant and diabetic target proteins. The docking studies were carried out for phytoligands using the iGEMDOCK program to elucidate the binding affinities to the target proteins. The binding interaction and conformation of each phytocompound with each target protein were predicted and ranked based on the iGEMDOCK-computed lowest energy and total binding energy, respectively. The best binding conformation for each phytocompound with each target protein was determined, if it showed the lowest total binding energy among its different conformations (Table 6).

In general, among the 25 phytocompounds, serverogenin acetate ([3,12-diacetyloxy-10,14-dimethyl-13-oxo15-(5-oxo-2H-furan-3-yl)-2-oxapentacyclo[9.7.0.01,3.05,10.014,18]octadecan-7-yl] acetate) exhibited the best binding conformations with the lowest binding energy values with bacterial $(-103.14 \mathrm{kcal} / \mathrm{mol})$, fungal $(-105.78 \mathrm{kcal} / \mathrm{mol})$, viral $(-90.53 \mathrm{kcal} / \mathrm{mol})$, antioxidant $(-82.85 \mathrm{kcal} / \mathrm{mol})$ and diabetic [e.g. aldose reductase $(-137.03 \mathrm{kcal} / \mathrm{mol})$, a-glucosidase $(-94.64 \mathrm{kcal} / \mathrm{mol})$ and $\alpha$-amylase $(-94.77 \mathrm{kcal} / \mathrm{mol})]$ target proteins (Table 6). Our findings are supported by the results of a previous study that showed that the lower the binding energy score was found the better the protein-ligand binding stability was identified ${ }^{24}$.

The serverogenin acetate formed the most excellent ligand-protein complexes (e.g. with six of seven tested target proteins) compared with other compounds. Next, docking analysis was then carried out for serverogenin acetate with the target proteins through AutoDock program. According to the binding energies, the docking 


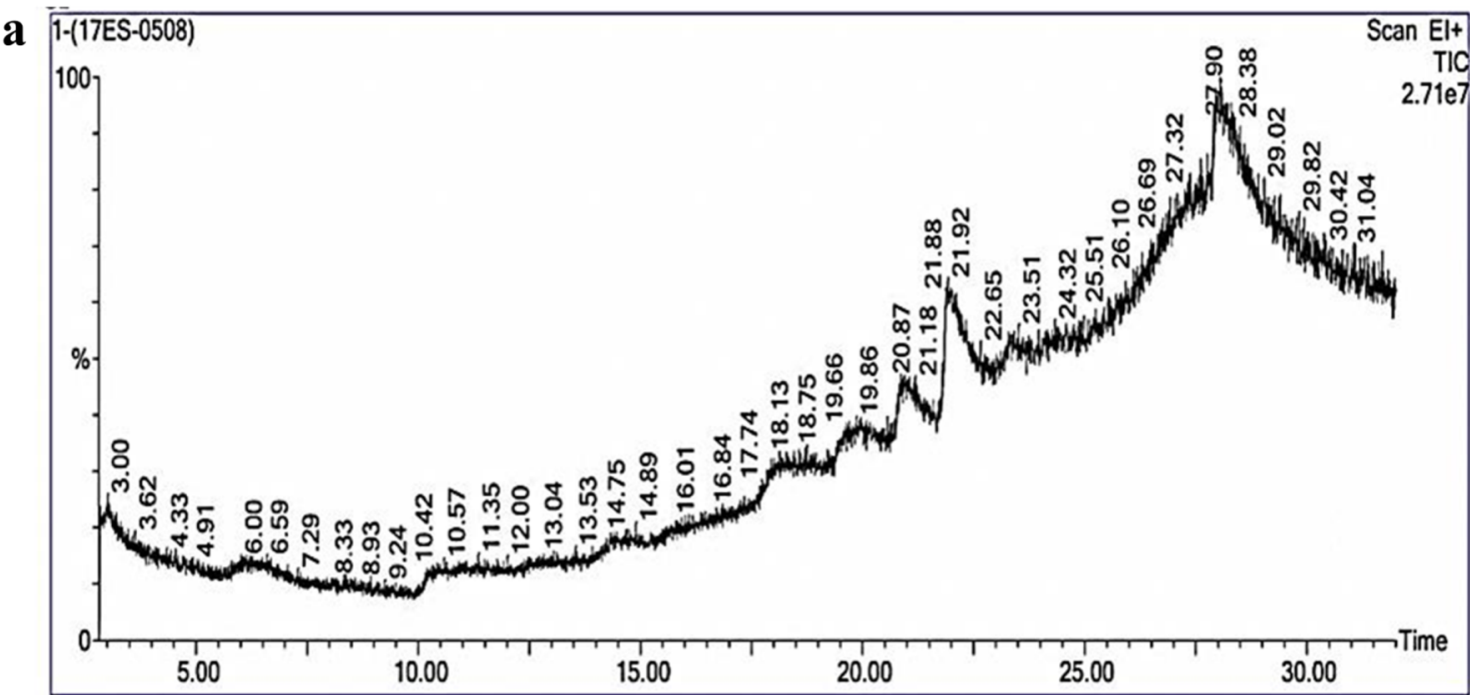

Inst() ACQUISITION PARAMETERS

Oven: Initial temp $60^{\circ} \mathrm{C}$ for $2.80 \mathrm{~min}$, ramp $10^{\circ} \mathrm{C} / \mathrm{min}$ to $300^{\circ} \mathrm{C}$, hold $6 \mathrm{~min}$, InjAauto $=260^{\circ} \mathrm{C}$, Volume $=0 \mu \mathrm{L}$, Split=10:1, Carrier Gas $=$ He, Solvent Delay $=2.80 \mathrm{~min}$, Transfer Temp $=240^{\circ} \mathrm{C}$, Source Temp $=240^{\circ} \mathrm{C}$, Scan: 40 to $600 \mathrm{Da}$, Column $30.0 \mathrm{~m} \times 250 \mu \mathrm{m}$

b<smiles>CCCC(ON)C(=O)O</smiles><smiles>C1C2CC3OC1OC3C2</smiles><smiles>CC(C)(C)CC(C)(C)c1ccc(O[Si](C)(C)C)cc1</smiles><smiles>C[Si](C)(C)c1ccccc1[Si](C)(C)C</smiles><smiles>C[Si](C)(C)c1ccc(=O)cc([Si](C)(C)C)c1</smiles><smiles>C[Si](C)(C)O</smiles><smiles>CC(CC(C)(C)c1ccccc1)c1ccc(O[Si](C)(C)C)cc1</smiles><smiles>CCC(c1ccc(O[Si](C)(C)C)cc1)C(CC)c1ccc(O[Si](C)(C)C)cc1</smiles><smiles>C[Si](C)(C)c1ccc([Si](C)(C)C)cc1</smiles><smiles>C[SiH](C)O[SiH](C)O[Si](C)(C)C</smiles>

Figure 2. (a) Phytoconstituents detected in the methanol leaf extract of Amomum nilgiricum using gas chromatography-mass spectrometry. (b). chemical structure of nine phytocompounds identified based on the retention time and peak area, namely (1) 5 -aminooxypentanoic acid (20.986 retention time and $4.082 \%$ peak area), (2) 3,7,9-trioxatricyclo[4.2.1.0 $0^{2,4}$ ]nonane (21.916 retention time and $19.379 \%$ peak area), (3) 1-(2,4,4-trimethylpentan2-yl)-4-[4-(2,4,4-trimethylpentan-2-yl)phenoxy]benzene (27.368 retention time and 3.129\% peak area), (4) trimethyl-(2-trimethylsilylphenyl)silane (27.753 retention time and $3.171 \%$ peak area), (5) 3,5-bis(trimethylsilyl) cyclohepta-2,4,6-trien-1-one, (27.898 retention time and 4.369\% peak area), (6) trimethyl-[4-[2-methyl-4(4-trimethylsilyloxyphenyl)pent-4-en-2-yl]phenoxy]silane (28.043 retention time and $38.631 \%$ peak area), (7) trimethyl-[4-[4-(4-trimethylsilyloxyphenyl)hexan-3- (28.288 retention time and $16.246 \%$ peak area), (8) trimethyl-[[4-(trimethylsilylmethyl)phenyl]methyl]silane (28.489 retention time and $6.405 \%$ peak area) and (9) $1,1,1,3,5,5,5$-heptamethyltrisiloxane (28.589 retention time and $4.588 \%$ peak area). 
a

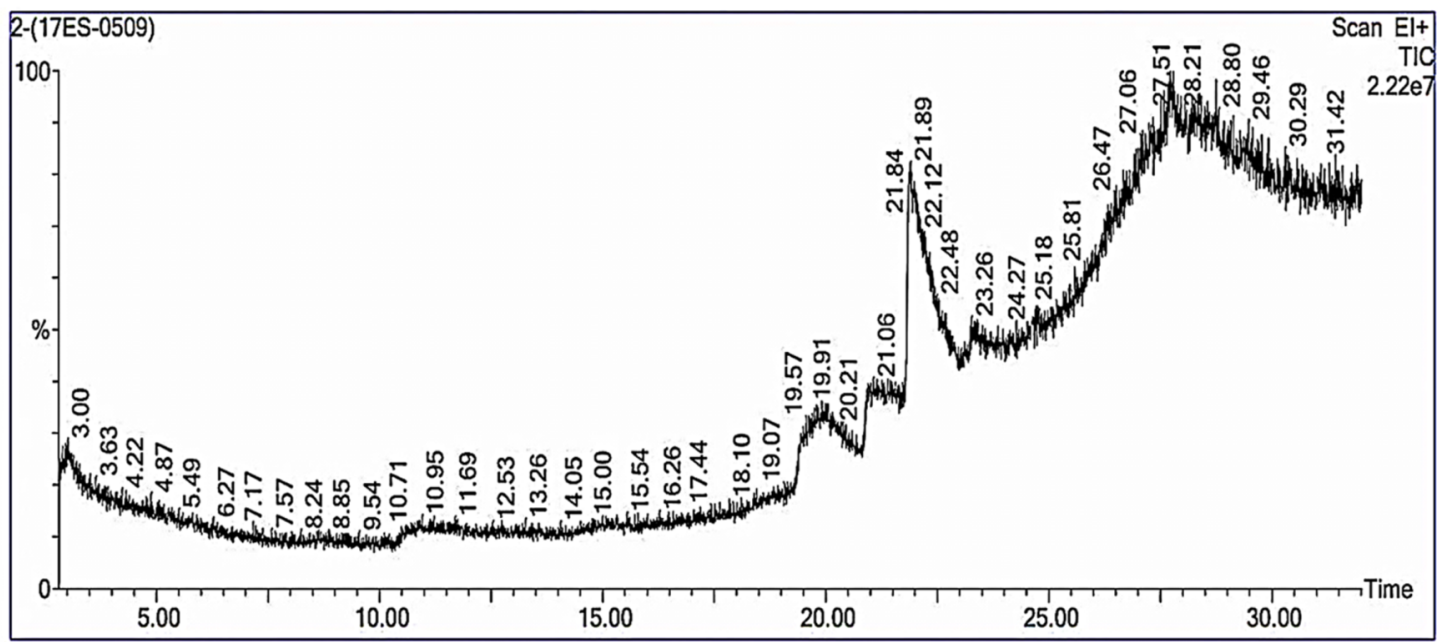

Inst() ACQUISITION PARAMETERS

Oven: Initial temp $60^{\circ} \mathrm{C}$ for $2.80 \mathrm{~min}$, ramp $10^{\circ} \mathrm{C} / \mathrm{min}$ to $300^{\circ} \mathrm{C}$, hold $6 \mathrm{~min}$, InjAauto $=260^{\circ} \mathrm{C}$, Volume $=0 \mu \mathrm{L}$, Split=10:1, Carrier Gas $=\mathrm{He}$, Solvent Delay $=2.80 \mathrm{~min}$, Transfer Temp $=240^{\circ} \mathrm{C}$, Source Temp $=240^{\circ} \mathrm{C}$, Scan: 40 to $600 \mathrm{Da}$, Column $30.0 \mathrm{~m} \times 250 \mu \mathrm{m}$

b 1

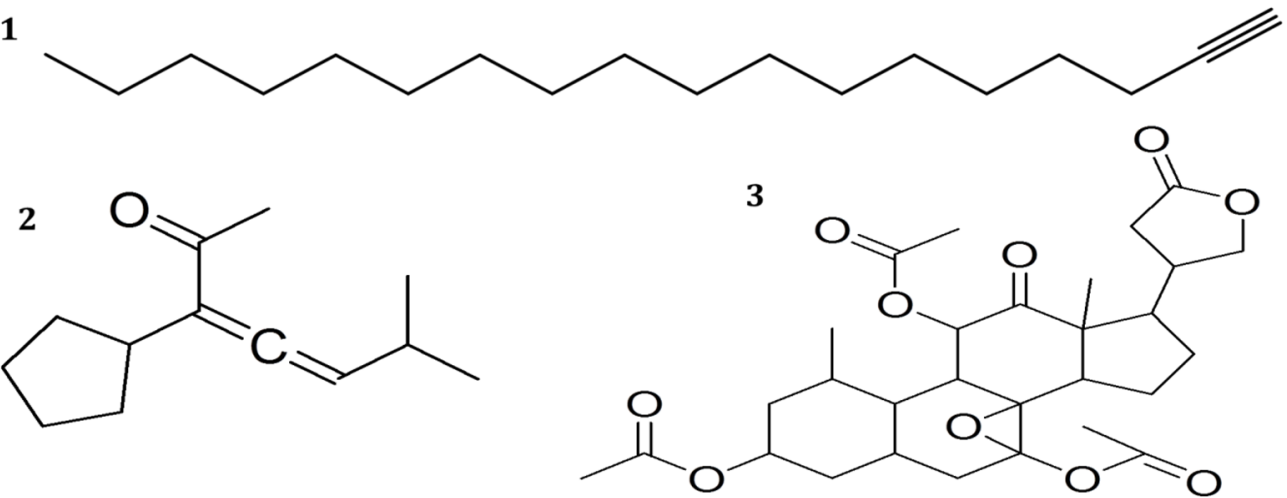<smiles>CC(C)(C)CC(C)(C)c1ccc(O[Si](C)(C)C)cc1</smiles><smiles>C[Si](C)(C)c1ccccc1[Si](C)(C)C</smiles><smiles>C[Si](C)(C)c1ccc(=O)cc([Si](C)(C)C)c1</smiles>

Figure 3. (a). Phytoconstituents detected in the ethyl acetate extract from Amomum nilgiricum leaves using gas chromatography-mass spectrometry. (b). Chemical structure of six phytocompounds identified based on the retention time and peak area, namely (1) octadec-1-yne, (19.950 retention time and $2.852 \%$ peak area), (2) 3,4-heptadien-2-one, 3-cyclopentyl-6-methyl- (21.086 retention time and 3.027\% peak area), (3) [3,12-diacetyloxy-10,14-dimethyl-13-oxo-15-(5-oxo-2H-furan-3-yl)-2-oxapentacyclo[9.7.0.01,3.05,10.014,18] octadecan-7-yl] acetate (21.891 retention time and $82.105 \%$ peak area), (4) trimethyl-[4-(2,4,4-trimethylpentan2-yl)phenoxy]silane (27.098 retention time and 3.757\% peak area), (5) trimethyl-(2-trimethylsilylphenyl) silane (27.718 retention time and 4.706\% peak area) and (6) 3,5-bis(trimethylsilyl)cyclohepta-2,4,6-trien-1-one ( 27.783 retention time and $3.553 \%$ peak area). 


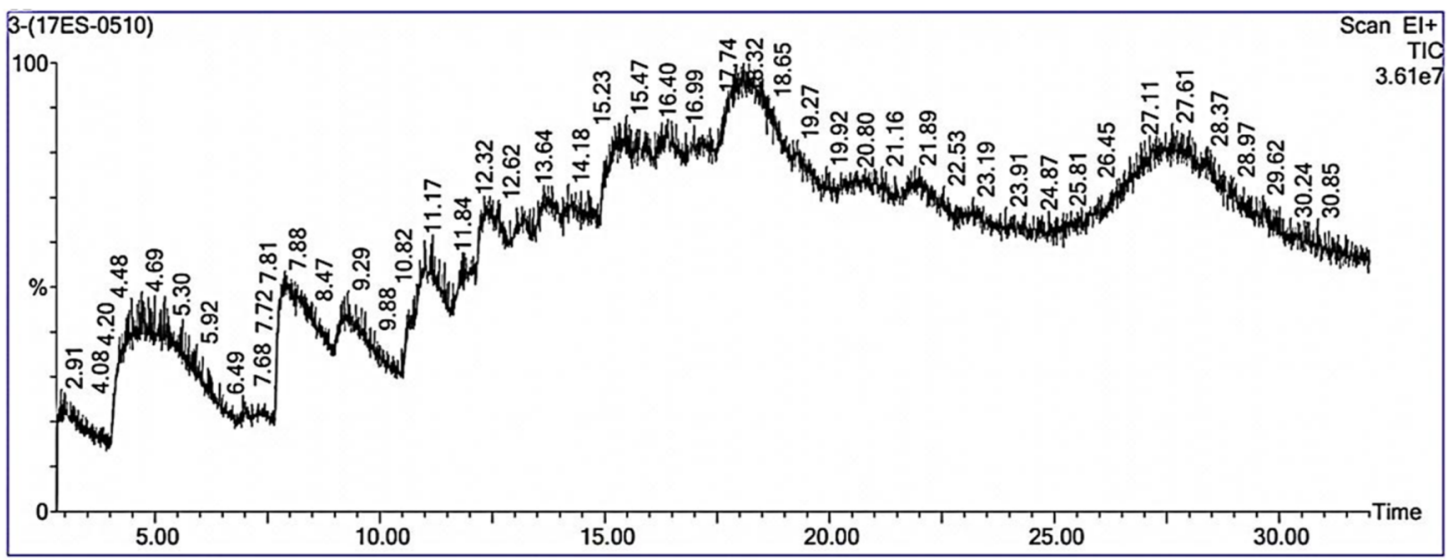

Inst() ACQUISITION PARAMETERS

Oven: Initial temp $60^{\circ} \mathrm{C}$ for $2.80 \mathrm{~min}$, ramp $10^{\circ} \mathrm{C} / \mathrm{min}$ to $300^{\circ} \mathrm{C}$, hold $6 \mathrm{~min}$, InjAauto $=260^{\circ} \mathrm{C}$, Volume $=0 \mu \mathrm{L}$, Split=10:1, Carrier Gas $=\mathrm{He}$, Solvent Delay $=2.80 \mathrm{~min}$, Transfer Temp $=240^{\circ} \mathrm{C}$, Source Temp $=240^{\circ} \mathrm{C}$, Scan: 40 to $600 \mathrm{Da}$, Column $30.0 \mathrm{~m} \times 250 \mu \mathrm{m}$

b 1<smiles>CCOC(=O)C(C)=O</smiles><smiles>O=C(O)CC(=O)O</smiles>

3<smiles>CCCCCCCCCCCCCCCC1OBOCC1N</smiles><smiles>CCCCS</smiles><smiles>Nc1c(N=O)[nH]c(=O)[nH]c1=O</smiles>

6<smiles>OC1COC2OC1O2</smiles>
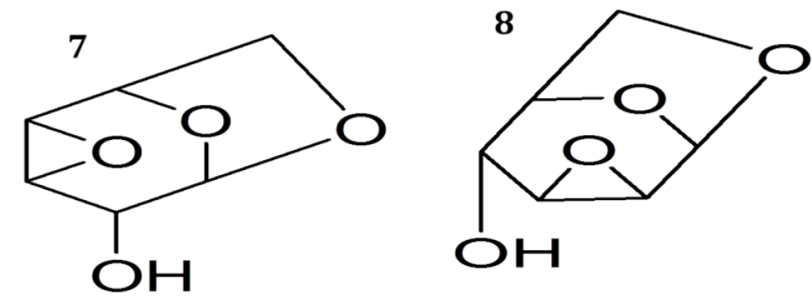

9

10

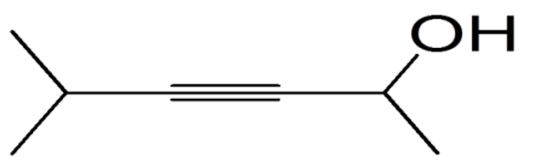<smiles>CCCCCCCCCCCCCCCCCCCCCCCC(=O)O[Si](C)(C)C</smiles>

Figure 4. (a). Phytoconstituents detected in the methanol rhizome extract of Amomum nilgiricum using gas chromatography-mass spectrometry. (b). Chemical structure of ten phytocompounds identified based on the retention time and peak area, namely (1) ethyl 2-oxopropanoate (4.759 retention time and $2.271 \%$ peak area), (2) $\left(1,3-{ }^{13} \mathrm{C}_{2}\right)$ propanedioic acid (7.880 retention time and $6.142 \%$ peak area), (3) 2-butyl-4-[(E)-pentadec-4enyl]-1,3,2-dioxaborinan-5-amine (15.473 retention time and $18.518 \%$ peak area), (4) 2,4-dimethyl-1,3-dioxane (16.399 retention time and $12.222 \%$ peak area), (5) 5-amino-6-nitroso-1 $H$-pyrimidine-2,4-dione (16.989 retention time and 9.325\% peak area), (6) (2R,4S,5S,6R)-3,8,9-trioxatricyclo[4.2.1.0 $0^{2,4}$ nonan-5-ol (18.084 retention time and $33.323 \%$ peak area), (7) $(1 R, 2 S, 4 R, 5 R)-3,7,9$-trioxatricyclo[4.2.1.0.4. retention time and $3.300 \%$ peak area), (8) $(2 S, 4 S, 5 R, 6 R)-3,8,9$-trioxatricyclo[4.2.1.0 $\left.0^{2,4}\right]$ nonan-5-ol (18.755 retention time and $9.621 \%$ peak area), (9) 5-methylhex-3-yn-2-ol (19.430 retention time and $2.818 \%$ peak area) and (10) trimethylsilyl tetracosanoate (21.911 retention time and $2.460 \%$ peak area). 


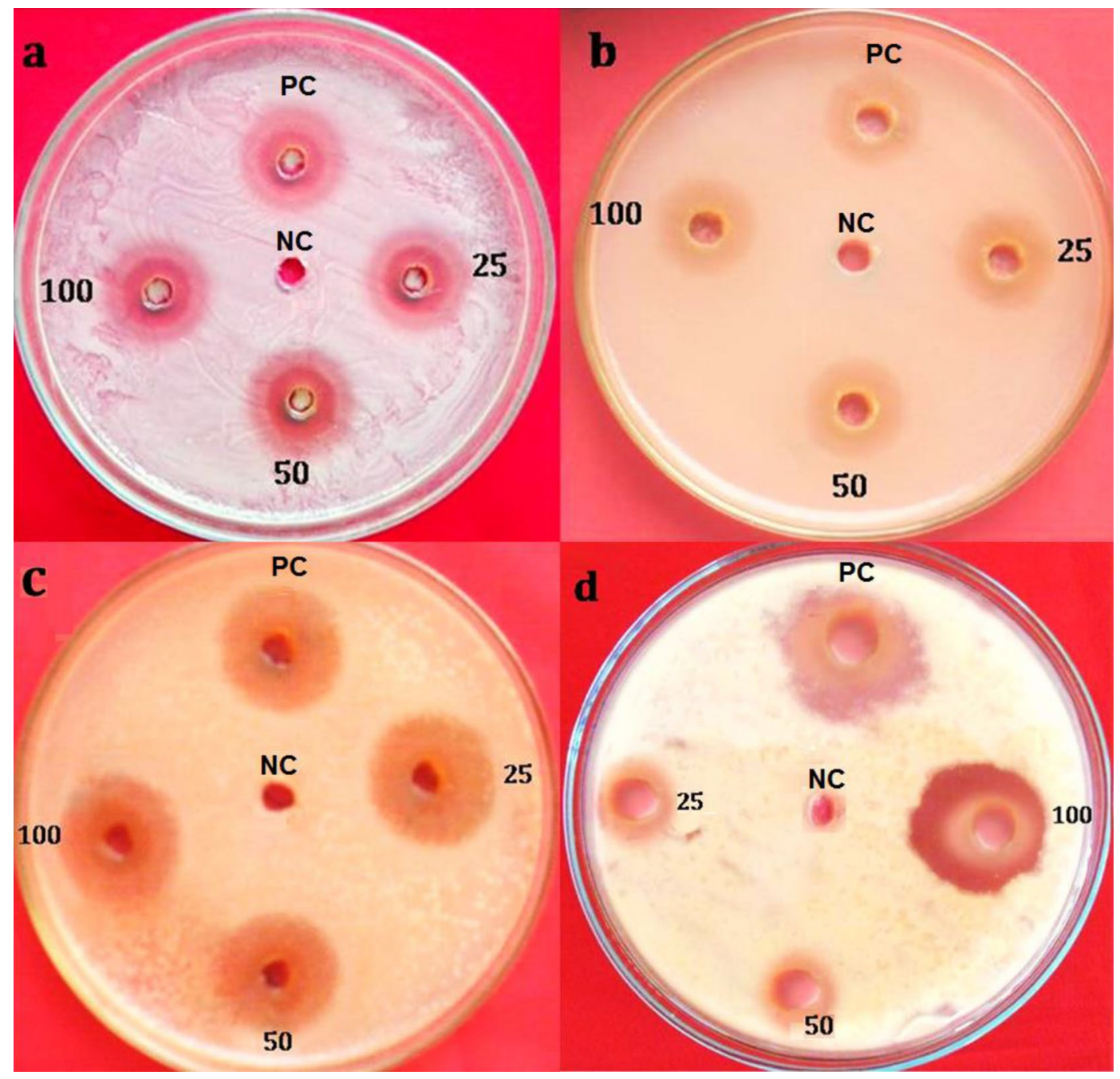

Figure 5. Antibacterial activity of leaf and rhizome extracts of A. nilgiricum at 25,50 and $100 \mathrm{mg} / \mathrm{mL}$ (a) Zone of inhibition from ethyl acetate leaf extract against Pseudomonas aeruginosa (b) Zone was from methanol leaf extract against Pseudomonas aeruginosa. (c) Zone of inhibition from ethyl acetate leaf extract against $R$. solanacearum (d) Zone of inhibition from methanolic rhizome extract against Ralstonia solanacearum. PC: Streptomycin (positive control); NC: solvent extract (negative control).

results of serverogenin acetate with target proteins were ranked. Analysis of the docking results showed the potential strength of binding affinity of serverogenin acetate into the binding sites of target proteins with minimum binding energies (ranging from -3.31 to $-4.22 \mathrm{kcal} / \mathrm{mol}$ ), ligand efficiency $(-0.08$ to $-0.11 \mathrm{kcal} / \mathrm{mol}$ ), inhibition constant (1.01 to $804.76 \mu \mathrm{M}$ ) and Van der Waals (VDW) + hydrogen bonding (Hbond) + desolvation energy $(-4.17$ to $-5.14 \mathrm{kcal} / \mathrm{mol}$ ) (Table 7). This serverogenin compound also formed hydrogen-bond interactions and the best possible binding pose with the residues of bacterial (Fig. 9a-c), fungal (Fig. 10a-c), viral (Fig. 11ac), antioxidant (Fig. 12a-c), aldose reductase (Fig. 13a-c), a-glucosidase (Fig. 14a-c) and a-amylase (Fig. 15a-c) target proteins, respectively as shown by their corresponding $3 \mathrm{D}$ interaction models.

\section{Discussion}

In the present study, the investigation of ethyl acetate and methanol extracts from leaves and methanol extract from rhizomes of Amomum nilgiricum revealed the presence of various phytoconstituents, including flavonoids, carbohydrates, anthocyanin, cardiac glycosides, tannins, phenols, amino acids, alkaloids, anthroquinone, steroids, proteins, terpenoids, leucoanthocyanin, phytosterols, saponins and diterpenes. These bioactive phytoconstituents could be responsible for the therapeutic ability of various extracts of $A$. nilgiricum. The analysis was carried out by gas chromatography-mass spectrometry (GC-MS), one of the most widely used techniques for separation of phytoconstituents. The GC-MS investigation of A. nilgiricum leaves and rhizome extracts revealed the presence of 25 phytochemical compounds, which could contribute to the medicinal properties of this plant species ${ }^{16,25}$. 5-(aminooxy)pentanoic acid has been reported to be present in ethanol stem and leaf extracts of Saccharum munja exhibiting antioxidant and antibacterial activities ${ }^{26}$. The phytocompound 3,7,9-trioxatricyclo[4.2.1.0 $\left.0^{2,4}\right]$ nonane extracted from methanol seeds extract of red Vitis vinifera has been reported for its antimicrobial and 


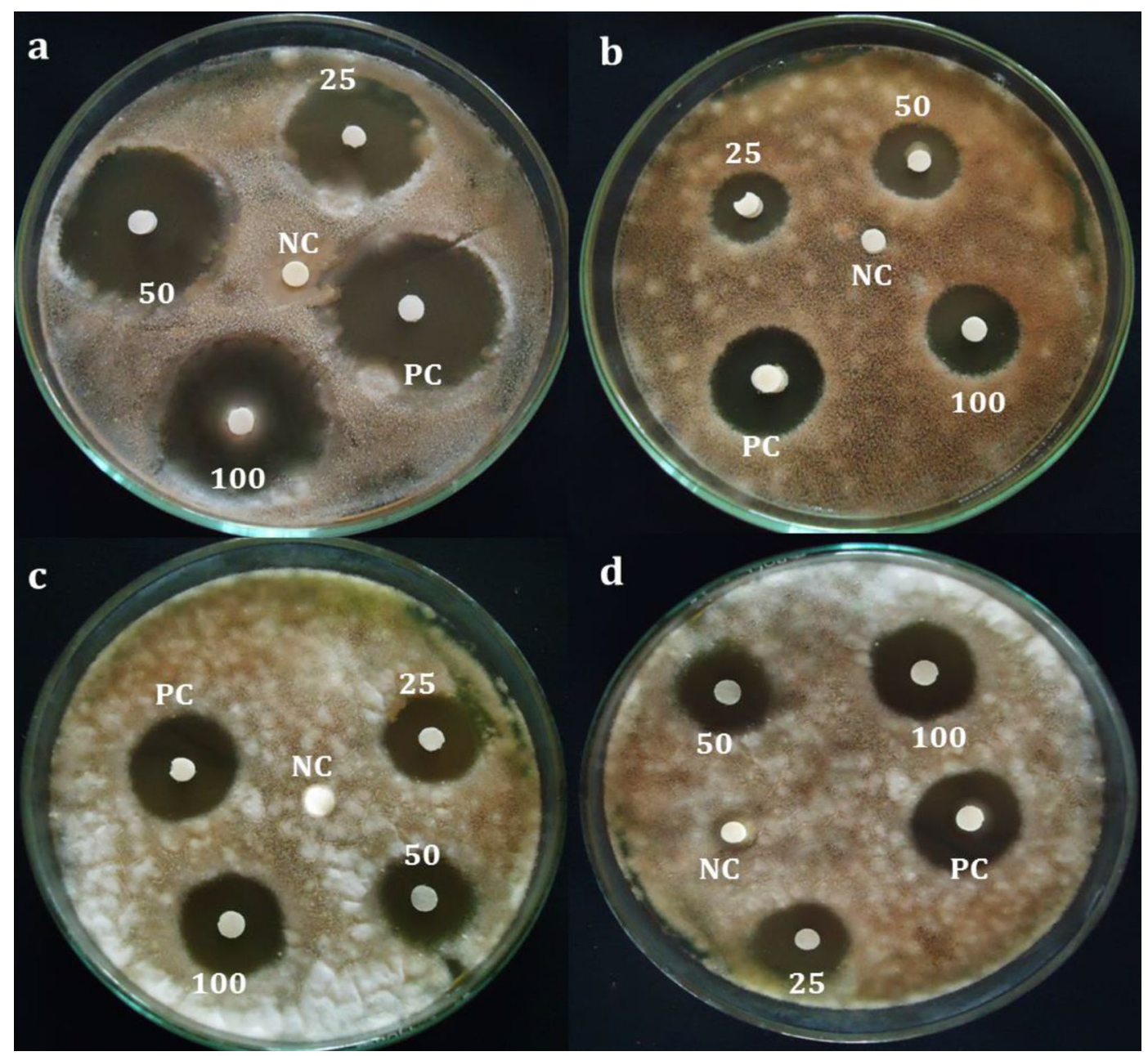

Figure 6. Antifungal activity of Amomum nilgiricum leaf and rhizome extracts at 25, 50 and $100 \mathrm{mg} / \mathrm{mL}$ (a) Zone of inhibition from ethyl acetate leaf extract against Alternaria alternata (b) methanol leaf extract against Alternaria alternata. (c) Ethyl acetate leaf extract against Pyricularia oryzae. (d) Methanolic rhizome extract against Pyricularia oryzae. PC: (Positive control) Nystatin; NC: Negative control (solvent).

anti-inflammatory activities ${ }^{27}$. The trimethyl-(2-trimethylsilylphenyl)silane is an aromatic hydrocarbon, and has been reported in aqueous extract of Centella asiatica displayed anticancer, antioxidant and antimicrobial activities ${ }^{28}$.

The cyclic compounds are unsaturated; and hence, play a key role in the antioxidant defense. 3,5-bis(trimethylsilyl)cyclohepta-2,4,6-trien-1-one is a ketone compound with antioxidant activity ${ }^{29}$, which has been isolated from methanol leaf extract of Psidium guajava and methanol leaf extract of Syzygium alternifolim ${ }^{30}$. The phytoconstituent 3,5-bis(trimethylsilyl)cyclohepta-2,4,6-trien-1-one which was identified from n-hexane seeds extract of Garcinia kola ${ }^{31}$ and ethanol leaf extract of Aporosa lindleyana ${ }^{32}$ so far there is no evidence of its biological activity ${ }^{30}$. Likewise, trimethyl-[[4-(trimethylsilylmethyl)phenyl]methyl]silane is an aromatic silica compound, reported from Cassia auriculata and Cardiospermum halicacabum ethanol leaf extracts ${ }^{33}$, however its biological activity has not yet documented ${ }^{33}$. On the other hand, phytocompound trimethyl-[4-[2-methyl4-(4-trimethylsilyloxyphenyl)pent-4-en-2-yl]phenoxy]silane was previously isolated from ethanol leaf extract of Wedelia trilobata ${ }^{34}$, trimethyl-[4-[4-(4-trimethylsilyloxyphenyl)hexan-3-yl]phenoxy] silane a sterol compound isolated from solvent mixture of chloroform and water (6:4) leaf and stem extracts of Paganum harmala ${ }^{35}$ and hexane and acetone (8:2) seeds extract of Butea monosperma ${ }^{36}$ documented with broad spectrum of antimicrobial properties $^{34-36}$. Similarly, 1,1,1,3,5,5,5-heptamethyltrisiloxane is a siloxane compound which has been isolated from methanol leaf extract of C. italica ${ }^{37}$, aqueous bark extracts of Khaya grandifoliola and Enantia chlorantha ${ }^{38}$ as well as from ethanol rhizome extract of Dryopteris cochleat ${ }^{39}$ exhibited anti-inflammatory and antimicrobial properties ${ }^{38,39}$. While, octadec-1-yne, an alkene compound has been reported from chloroform extract of Spermadictyon suaveolens flowers showed antibacterial activity ${ }^{40}$. On contrary, octadec-1-yne extracted from petroleum ether and ethyl acetate leaves extracts of Leucaena leucocephala from Malaysia ${ }^{41}$ did not possess any phytopharmaceutical properties. In an independent study, the monoterpeniod compound, 3,4-heptadien-2-one, 3-cyclopentyl-6-methyl- reported from hexane flower extract of Acmella uliginosa ${ }^{42}$ and from hydrophilic leaf and flower extracts of Succisa pratensis ${ }^{43}$ reported significant antibacterial and antifungal activities. 


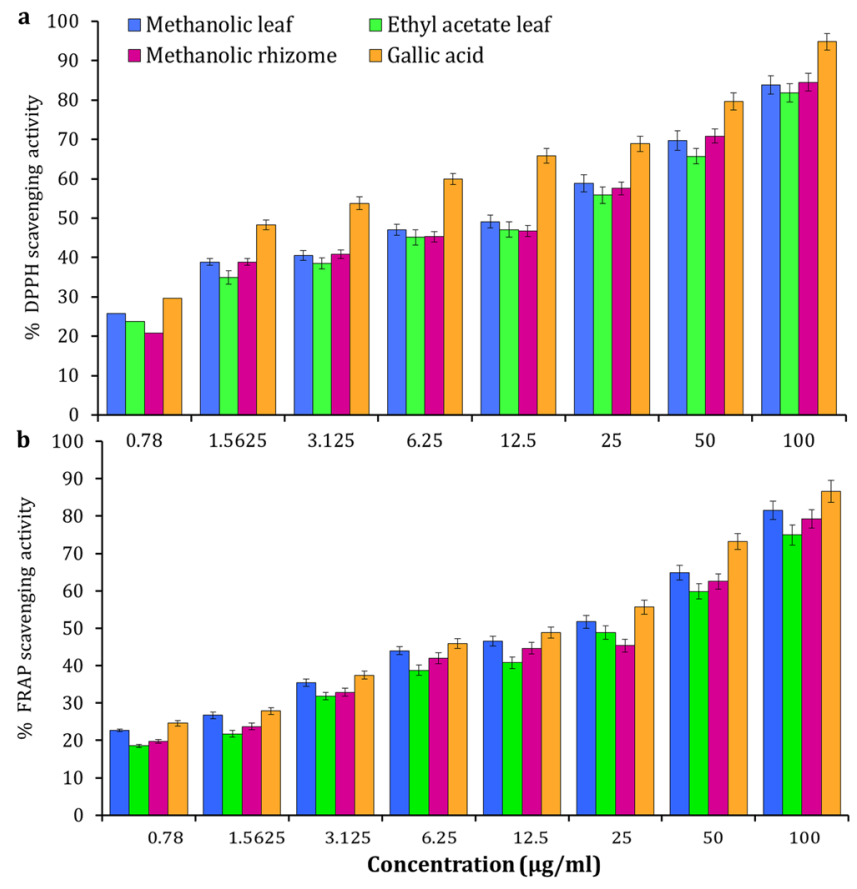

Figure 7. Antioxidant activity of Amomum nilgiricum leaf and rhizome extracts. (a) DPPH radical scavenging activity. (b) Ferric reducing antioxidant power scavenging activity.

Serverogenin acetate is a bioactive compound known for its application in pharmacology, this bioactive compound isolated from methanol leaf extracts of Trichilia connaroides demonstrated broad spectrum of biological properties such as antioxidant, anti-insect, antimicrobial, anticancer and antiulcerogenic activities ${ }^{44}$. The trimethylsilyl derivative compound trimethyl-[4-(2,4,4-trimethylpentan-2-yl)phenoxy]silane identified from n-hexane seed extract of G. kola ${ }^{31}$, chloroform and ethanol leaf extracts of Acacia karroo and Ziziphus mauritian ${ }^{45}$ produced significant antibacterial activity against clinical bacteria and also recorded anti-inflammatory, anticancer, analgesic and diuretic activities. Ethyl 2-oxopropanoate is an androstane natured compound reported from methanol leaves extract of $A$. nilotica ${ }^{46}$ and Kamettia caryophyllat ${ }^{47}$ showed potent bioactivities of acidifier, acidulant and inhibits production of uric acid ${ }^{47} \cdot\left(1,3-{ }^{13} \mathrm{C}_{2}\right)$ propanedioic acid has been isolated from methanol extract of Alysicarpus monilifer whole plant ${ }^{48}$ however its biological activity is yet to confirm. The flavonoid compound 2,4-dimethyl-1,3-dioxane known to possess anti-inflammatory, analgesic, antibacterial and antifungal activities was isolated from Arjunarishta, a modified ayurvedic medicine ${ }^{49}$. 5-amino-6-nitroso$1 H$-pyrimidine-2,4-dione from methanol leaf extract of Stachytarpheta jamaicensis capable of producing high multi therapeutic properties such as antioxidant, anti-arthritic, anti-inflammatory and bactericidal potentials ${ }^{50}$. $(2 S, 4 S, 5 R, 6 R)-3,8,9$-trioxatricyclo[4.2.1. $\left.0^{2,4}\right]$ nonan-5-ol is a sugar moiety natured compound identified from ethanol extract of aerial parts of Crotalaria longipes ${ }^{51}$ and ethanol extracts of whole plant of Sarcostemma seca$m o n e^{52}$ reported to be used as a preservative. In this study, the leaf and rhizome extracts of $A$. nilgiricum showed remarkable in vitro antibacterial and antifungal activities by suppressing their colony and growth rate. In support of this study, earlier our group reported the potential antimicrobial activities of rhizome extracts of $A$. niligiricum $^{53}$. In addition we have also noticed remarkable antioxidant and antidiabetic activities from the leaf and rhizome extracts of $A$. nilgiricum which is the first kind of report from this study. Previously, the antibacterial and antioxidant activities using the essential oil extracted from A. subulatum was documented Shrestha ${ }^{54}$. Similarly, Sharma et al. ${ }^{55}$ reported the antioxidant activity by seed extract of A. subulatum.

From the above evidences, it can be elucidated that $A$. nilgiricum plant consists of enormous potential of pharmacological constituents, therapeutic phytocompounds responsible for various pharmacological actions like antimicrobial, antioxidant anti-inflammatory, antidiabetic, analgesic, antiaging, anticancer, hepatoprotective, hypercholesterolemic, antihistaminic, antiandrogenic, antifibrinolytic, diuretic, antiasthma activities, preservative etc. These major chemical compounds identified from different crude extracts are considered to be a part of plants' defense systems and they may be grouped as protective compounds found in this plant referring to them as 'phytoanticipins' and 'phytoprotectants ${ }^{56}$. Thus, the identification of a various phytochemical compounds from ethyl acetate and methanol extracts from leaves and rhizomes of $A$. nilgiricum display significant medicinal properties of the plant $A$. nilgiricum. Further studies like bio-prospecting are essential to support its biological properties and biological importance of these innovative bio-molecules will be interesting to be studied. To the best of our knowledge, this is the first report on GC and MS investigation of A. nilgiricum leaf and rhizome extracts.

Bioinformatics tools provide a great support to pharmaceutical companies in the process of drug discovery in a short duration of time with less cost using all primary information obtained from in vivo and in vitro analysis. In silico molecular docking in one of the greatest methods to determine new ligand for proteins of identified 
a

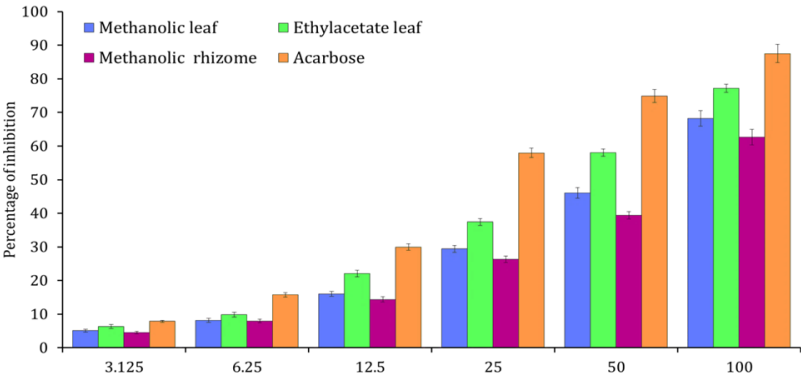

b
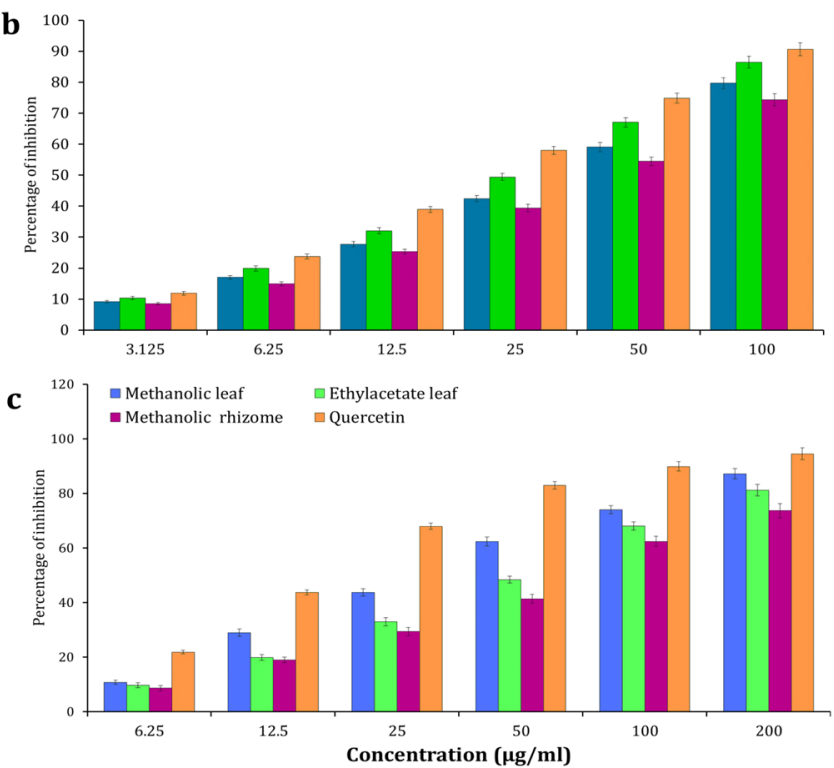

Figure 8. Antidiabetic enzymes activities of Amomum nilgiricum leaf and rhizome extracts. (a) $\alpha-$ amylase inhibition activity. (b) a-glucosidase inhibition activity. (c) aldose reductase inhibition activity.

structure and thus play an important role in structure based drug discovery. Investigators worldwide use computer docking programs to discover and investigate the binding affinity for compounds that fit a binding site on the protein. The structure of the protein and ligand should be three dimensional ${ }^{57}$. The study and documentation of structural compounds from different medicinal plant species are gaining interest and importance. In the structure based drug design, molecular docking is generally used to predict and inter molecular complex between the drug compounds with its target protein ${ }^{58}$. In the present study, a total of 25 bioactive compounds were identified from A. nilgiricum leaf and rhizome extracts by GC-MS analysis and used for molecular docking studies. Further, the compounds were analyzed for bioactivity against the target proteins. Serverogenin acetate is the lead compound and exhibited antibacterial, antifungal, antiviral, antioxidant and antidiabetic activities. Computational simulation studies revealed that serverogenin acetate compound recorded better affinity with low binding energy in comparison with other compounds. The docking study results also showed that various energy sources are consistent and contribute to the overall strength to the binding interactions of serverogenin acetate for each target proteins.

\section{Conclusions}

The present investigation was focused on identification of various bioactive compounds from the leaf and the rhizome extracts of Amomum nilgiricum for the first time by GC-MS analysis. These compounds are responsible for the different therapeutic and pharmacological properties. We have also provided the evidence of leaves and rhizome extracts of $A$. nilgiricum for its antimicrobial (phytopathogens), antioxidant and antidiabetic activities. The serverogenin acetate ([3,12-diacetyloxy-10,14-dimethyl-13-oxo-15-(5-oxo-2H-furan-3-yl)-2-oxapentacyclo[9.7.0.01,3.05,10.014,18]octadecan-7-yl] acetate) compound showed promising binding affinity toward different proteins in molecular docking experiments, and their drug-like features were demonstrated through the iGEMDOCK docking analysis. Using the serverogenin acetate compound may enable us to develop an effective drug against pathogenic bacteria, fungi and viruses, as well as diabetic diseases. Further investigations to determine its bioactivity, toxicity profile and clinical studies are necessary for broad-spectrum drug discovery. 


\begin{tabular}{|c|c|c|c|c|c|c|c|}
\hline \multirow[b]{2}{*}{ Name of the compound } & \multicolumn{7}{|l|}{ Target proteins } \\
\hline & $\begin{array}{l}\begin{array}{l}\text { Antibacterial } \\
\text { (kcal/mol) }\end{array} \\
\end{array}$ & $\begin{array}{l}\text { Antifungal (kcal/ } \\
\text { mol) }\end{array}$ & $\begin{array}{l}\text { Antiviral (kcal / } \\
\text { mol) }\end{array}$ & $\begin{array}{l}\text { Antioxidant (kcal/ } \\
\text { mol) }\end{array}$ & $\begin{array}{l}\text { Aldo reductase } \\
\text { (kcal/mol) }\end{array}$ & $\begin{array}{l}\text { Alpha glucosidase } \\
\text { (kcal/mol) }\end{array}$ & \begin{tabular}{|l} 
Alpha amylase \\
(kcal/mol)
\end{tabular} \\
\hline 5-aminooxypentanoic acid & -66.752 & -71.1418 & -69.0289 & -63.1516 & -68.1574 & -66.9723 & -71.7856 \\
\hline $\begin{array}{l}\text { 3,7,9-trioxatricyclo }\left[4 \cdot 2 \cdot 1 \cdot 0^{2,4}\right] \\
\text { nonane }\end{array}$ & -54.5983 & -57.2671 & -56.0637 & -53.3396 & -66.216 & -67.5377 & -57.9483 \\
\hline \begin{tabular}{|l|} 
1-(2,4,4-trimethylpentan- \\
2-yl)-4-[4-(2,4,4-trimethyl- \\
pentan-2-yl)phenoxy]benzene
\end{tabular} & -65.0987 & -68.3489 & -62.7397 & -57.2621 & -84.2382 & -77.612 & -66.8286 \\
\hline $\begin{array}{l}\text { Trimethyl-(2-trimethylsilyl- } \\
\text { phenyl)silane }\end{array}$ & -56.2474 & -55.4126 & -55.5996 & -52.3413 & -68.2982 & -59.2383 & -55.877 \\
\hline \begin{tabular}{|l|} 
3,5-bis(trimethylsilyl) \\
cyclohepta-2,4,6-trien-1-one
\end{tabular} & -72.7044 & -82.1708 & -68.961 & -75.8948 & -101.154 & -68.9778 & -72.3101 \\
\hline \begin{tabular}{|l|} 
Trimethyl-[4-[2-methyl-4-(4- \\
trimethylsilyloxyphenyl)pent- \\
4-en-2-yl]phenoxy]silane
\end{tabular} & -75.6489 & -73.6996 & -69.0353 & -66.5933 & -74.3881 & -75.7783 & -76.2131 \\
\hline \begin{tabular}{|l|} 
Trimethyl-[4-[4-(4-trimethyl- \\
silyloxyphenyl)hexan-3-yl] \\
phenoxy]silane
\end{tabular} & -46.8452 & -52.8639 & -45.2415 & -42.2495 & -53.1494 & -53.0229 & -45.8263 \\
\hline \begin{tabular}{|l|} 
Trimethyl-[[4- \\
(trimethylsilylmethyl)phenyl] \\
methyl]silane
\end{tabular} & -46.4648 & -49.5256 & -53.544 & -51.1354 & -59.5502 & -56.6869 & -49.4279 \\
\hline $\begin{array}{l}\text { 1,1,1,3,5,5,5-heptamethyltrisi- } \\
\text { loxane }\end{array}$ & -60.0582 & -57.4102 & -55.932 & -47.7934 & -57.7133 & -59.4908 & -55.2875 \\
\hline Octadec-1-yne & -65.6492 & -81.3123 & -74.2114 & -62.2128 & -85.6565 & -69.7406 & -59.9751 \\
\hline $\begin{array}{l}\text { 3,4-heptadien-2-one, } \\
\text { 3-cyclopentyl-6-methyl- }\end{array}$ & -60.6587 & -67.5671 & -67.3529 & -59.9746 & -67.1473 & -63.9103 & -61.7223 \\
\hline $\begin{array}{l}\text { Serverogenin acetate } \\
{[3,12 \text {-diacetyloxy-10,14-di- }} \\
\text { methyl-13-oxo-15-(5-oxo- } \\
\text { 2H-furan-3-yl)-2-oxapenta- } \\
\text { cyclo[9.7.0.01,3.05,10.014,18] } \\
\text { octadecan-7-yl] acetate }\end{array}$ & -103.145 & -105.789 & -90.5357 & -82.8571 & -137.034 & -94.6438 & -94.7775 \\
\hline \begin{tabular}{|l|} 
Trimethyl-[4-(2,4,4-trimethyl- \\
pentan-2-yl)phenoxy]silane
\end{tabular} & -65.0283 & -75.0271 & -66.8266 & -61.3058 & -88.5434 & -77.6173 & -66.588 \\
\hline $\begin{array}{l}\text { Trimethyl-(2-trimethylsilyl- } \\
\text { phenyl)silane }\end{array}$ & -55.7175 & -58.7707 & -56.8283 & -48.5849 & -59.4178 & -59.3893 & -55.4465 \\
\hline \begin{tabular}{|l|} 
3,5-bis(trimethylsilyl) \\
cyclohepta-2,4,6-trien-1-one
\end{tabular} & -53.0653 & -59.9694 & -58.4119 & -54.0375 & -64.6093 & -58.5245 & -59.7577 \\
\hline Ethyl 2-oxopropanoate & -62.6881 & -73.4001 & -46.0156 & -55.4678 & -69.9755 & -64.1391 & -69.1988 \\
\hline$\left(1,3-{ }^{13} \mathrm{C}_{2}\right)$ propanedioic acid & -74.3819 & -88.8806 & -83.2791 & -74.408 & -106.82 & -76.2029 & -86.8522 \\
\hline $\begin{array}{l}\text { 2-butyl-4-[(E)-pentadec- } \\
\text { 4-enyl]-1,3,2-dioxaborinan- } \\
\text { 5-amine }\end{array}$ & -49.5347 & -60.9228 & -47.6395 & -49.6682 & -56.4944 & -55.7986 & -48.7232 \\
\hline 2,4-dimethyl-1,3-dioxane & -75.7941 & -76.8513 & -77.4718 & -75.5358 & -87.5893 & -78.6818 & -81.3197 \\
\hline $\begin{array}{l}\text { 5-amino-6-nitroso-1H-pyrim- } \\
\text { idine-2,4-dione }\end{array}$ & -58.8103 & -62.1977 & -59.1507 & -62.4201 & -74.5919 & -69.2034 & -64.595 \\
\hline $\begin{array}{l}(2 R, 4 S, 5 S, 6 R)-3,8,9 \text {-trioxatri- } \\
\text { cyclo[4.2.1.0.2, }] \text { nonan-5-ol }\end{array}$ & -63.0445 & -64.2929 & -58.6933 & -64.2665 & -76.5104 & -77.3524 & -67.3094 \\
\hline $\begin{array}{l}(1 R, 2 S, 4 R, 5 R)-3,7,9 \text {-trioxatri- } \\
\text { cyclo[4.2.1. }\end{array}$ & -58.317 & -66.9296 & -57.4211 & -62.3883 & -74.6524 & -69.2782 & -64.748 \\
\hline $\begin{array}{l}(2 S, 4 S, 5 R, 6 R)-3,8,9 \text {-trioxatri- } \\
\text { cyclo[4.2.1.0 }\end{array}$ & -58.1171 & -58.2697 & -49.6619 & -53.7779 & -58.2695 & -59.8385 & -51.2265 \\
\hline 5-methylhex-3-yn-2-ol & -79.8391 & -85.5672 & -93.6498 & -62.3278 & -85.3802 & -88.8687 & -78.6849 \\
\hline \begin{tabular}{|l|} 
Trimethylsilyl tetracosanoate \\
\end{tabular} & -61.5327 & -59.2993 & -71.4039 & -60.1473 & -57.8295 & -79.5731 & -65.2117 \\
\hline
\end{tabular}

Table 6. Binding energies of 25 phytocompounds against bacterial, fungal, viral, antioxidant and diabetic (e.g. $\alpha$-glucosidase, $\alpha$-amylase and aldose reductase) target proteins.[3,12-diacetyloxy-10,14-dimethyl-13-oxo-15(5-oxo-2H-furan-3-yl)-2-oxapentacyclo[9.7.0.01,3.05,10.014,18]octadecan-7-yl] acetate (red) showed the best binding conformations with tested target proteins.

\section{Methods}

Collection and preparation of plant materials. The fresh leaves and rhizomes of Amomum nilgiricum (V.P. Thomas \& M. Sabu) were collected from Western Ghats region of Palakkad district Kerala, India $\left(11^{\circ} 03^{\prime}\right.$ $15.46^{\prime \prime} \mathrm{N}, 076^{\circ} 32^{\prime} 23.58^{\prime \prime} \mathrm{E}$ ) at altitude of $1150 \mathrm{~m}$ above mean sea level during 2017. The freshly collected leaves were thoroughly washed in running tap water and rinsed in distilled water before they were cut into small bits, then shade-dried at room temperature for 10 days, powdered using laboratory blender and preserved in $1000 \mathrm{ml}$ air tight bottle. The dry leaf powder samples (100 g/each) were individually extracted with ethyl acetate and 


\begin{tabular}{|l|l|l|l|l|}
\hline Target protein & Binding energy $(\mathbf{K c a l} / \mathbf{m o l})$ & Ligand efficiency $(\mathbf{K c a l} / \mathbf{m o l})$ & Inhibition constant $(\boldsymbol{\mu M})$ & $\begin{array}{l}\text { Van der Waals + hydrogen bonding + desolvation energy } \\
(\mathbf{K c a l} / \mathbf{m o l})\end{array}$ \\
\hline Antibacterial 5iwm & -4.22 & -0.11 & 804.76 & -4.9 \\
\hline Antifungal 4i9p & -4.09 & -0.1 & 1.01 & -5.14 \\
\hline Antiviral 1rev & -3.65 & -0.09 & 2.11 & -4.32 \\
\hline Antioxidant 2he3 & -3.31 & -0.08 & 3.75 & -4.17 \\
\hline Aldo reductase 4ys1 & -4.03 & -0.1 & 1.11 & -5.08 \\
\hline Alpha glucosidase 5nn3 & -4.03 & -0.1 & 1.11 & -4.79 \\
\hline Alpha amylase i-tasser & -3.82 & -0.1 & 1.58 & -4.56 \\
\hline
\end{tabular}

Table 7. Automated docking binding interactions results of serverogenin acetate ([3,12-diacetyloxy-10,14dimethyl-13-oxo-15-(5-oxo-2H-furan-3-yl)-2-oxapentacyclo[9.7.0.01,3.05,10.014,18] octadecan-7-yl] acetate) with target proteins.

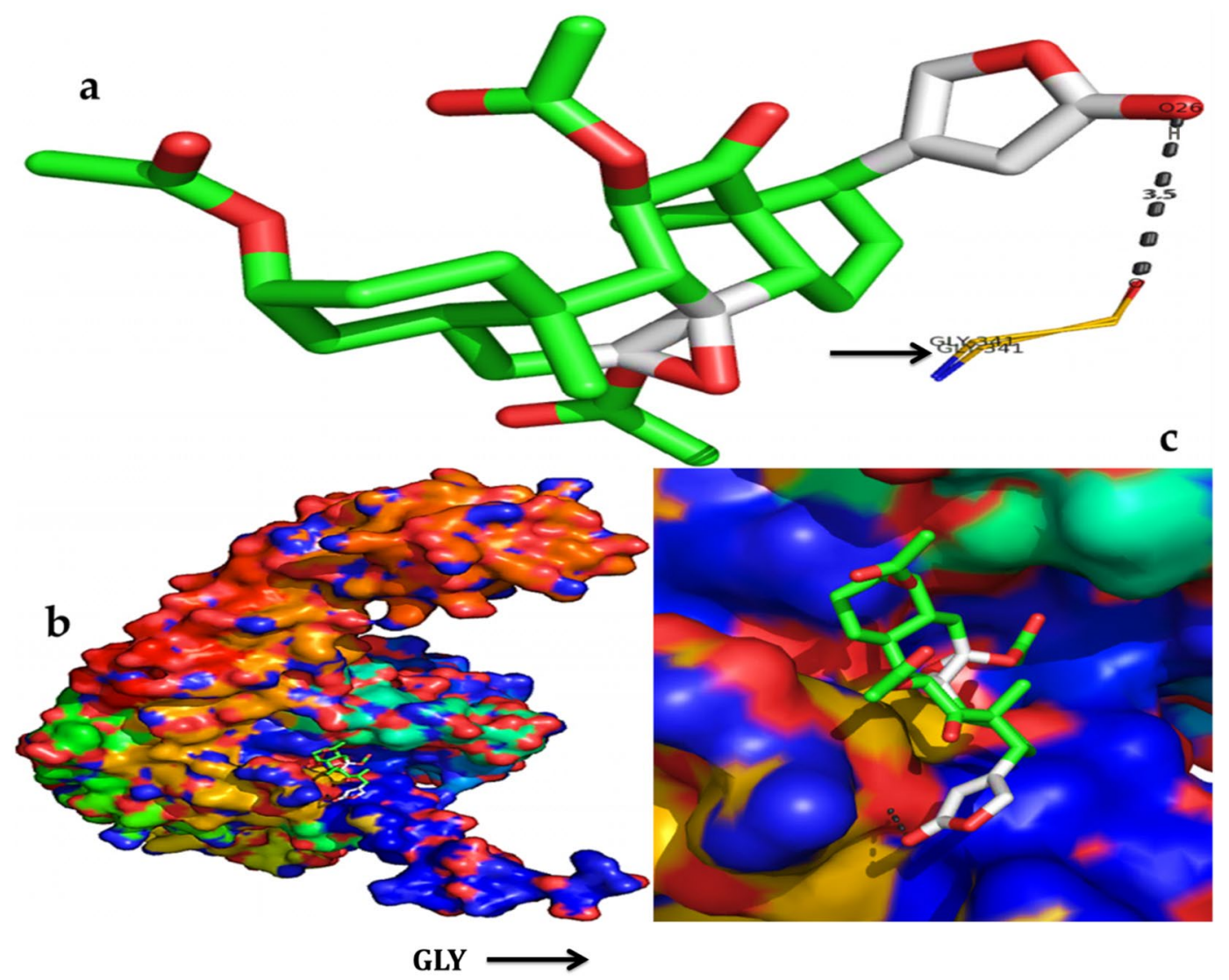

Figure 9. In silico molecular docking shows the binding interaction of serverogenin acetate ([3,12-diacetyloxy10,14-dimethyl-13-oxo-15-(5-oxo-2H-furan-3-yl)-2-oxapentacyclo[9.7.0.01,3.05,10.014,18] octadecan-7-yl] acetate) compound with bacterial target protein (5iwm) based on the binding energy generated by AutoDock program. (a) A hydrogen-bond interaction is formed by O26 in GLY341 (black arrow) in the serverogenin acetate compound. (b) A close-up view of serverogenin acetate compound that binds to the bacterial target protein. (c) Binding pose of the bacterial target protein with serverogenin acetate compound. 


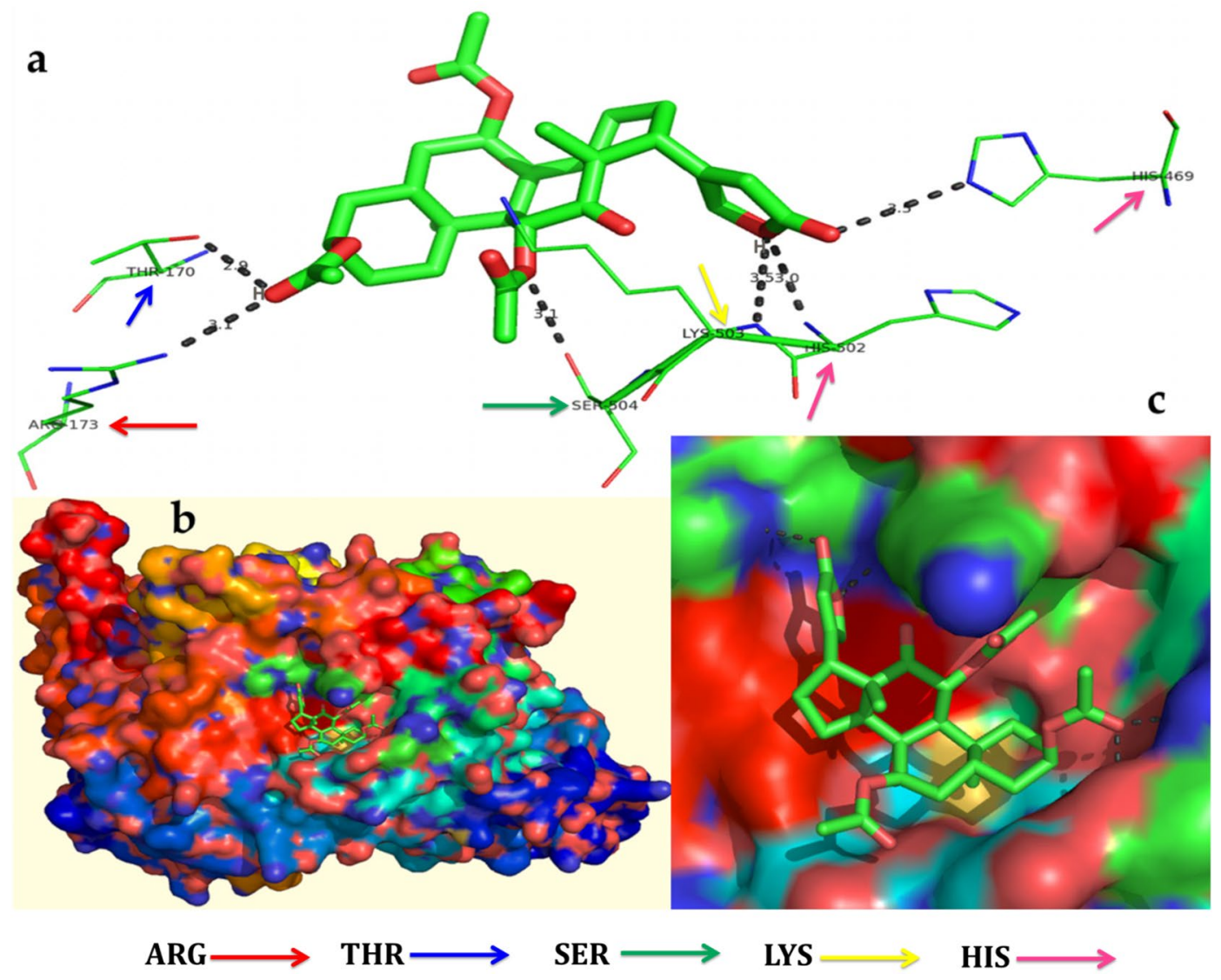

Figure 10. In silico molecular docking shows the binding interaction of serverogenin acetate ([3,12-diacetyloxy-10,14-dimethyl-13-oxo-15-(5-oxo-2H-furan-3-yl)-2-oxapentacyclo[9.7.0.01,3.05,10.014,18] octadecan-7-yl] acetate) compound with fungal target protein (4i9p) based on the binding energy generated by AutoDock program. (a) Hydrogen-bond interaction are formed by THR170 (blue arrow), ARG173 (red arrow), SER504 (green arrow), LYS503 (yellow arrow), HIS502 and HIS469 (pink arrow) in the serverogenin acetate compound. (b) A close-up view of serverogenin acetate compound that binds to the fungal target protein. (c) Binding pose of fungal target protein with serverogenin acetate compound.

methanol solvents $(500 \mathrm{~mL})$ in a Soxhlet apparatus for $8 \mathrm{~h}$ respectively at a temperature not beyond the boiling point of the solvents. The ethyl acetate and methanol extracts were filtered using Whatman No. 1 filter paper, and the filtrates were concentrated under reduced pressure with a rotary evaporator, freeze-dried in a lyophilizer, and then kept at $4{ }^{\circ} \mathrm{C}$. Similarly, the freshly collected rhizomes were thoroughly washed in running tap water and rinsed in distilled water. Subsequently, the samples were cut into small pieces, shade-dried at room temperature for 10 days, powered and extracted with methanol in a Soxhlet unit for $8 \mathrm{~h}$. The extracted samples were filtered by Whatman filter paper, and the filtrates were concentrated at reduced pressure with a rotary evaporator, dried using a lyophilizer and kept at $4{ }^{\circ} \mathrm{C}$ until further use.

Preliminary phytochemical screening. The qualitative phytochemical prescreening of leaf and rhizome extracts was performed following the previously published protocols ${ }^{59}$. The standard solution was prepared from $100 \mathrm{mg}$ leaf or rhizome extract by dissolving the extract in $10 \mathrm{~mL}$ ethyl acetate or methanol. These solutions were then screened for the presence of different phytochemicals; namely, phenols, carbohydrates, flavonoids, anthocyanin, cardiac glycosides, saponins, tannins, alkaloids, steroids, terpenoids, amino acids, leucoanthocyanin, anthroquinone, proteins, phytosterols and diterpenes.

Fourier transform infrared spectrometer (FTIR). The FT-IR was performed with resolution in the spectral area of $4000-400 \mathrm{~cm}^{-1}$ to detect the possible functional groups. $10 \mathrm{mg}$ of the dried A. nilgiricum leaf and rhizome extracts powder was encapsulated in $100 \mathrm{mg}$ of $\mathrm{KBr}$ salt pellet, using a mortar and pestle, compressed into a thin pellet ${ }^{13}$. 


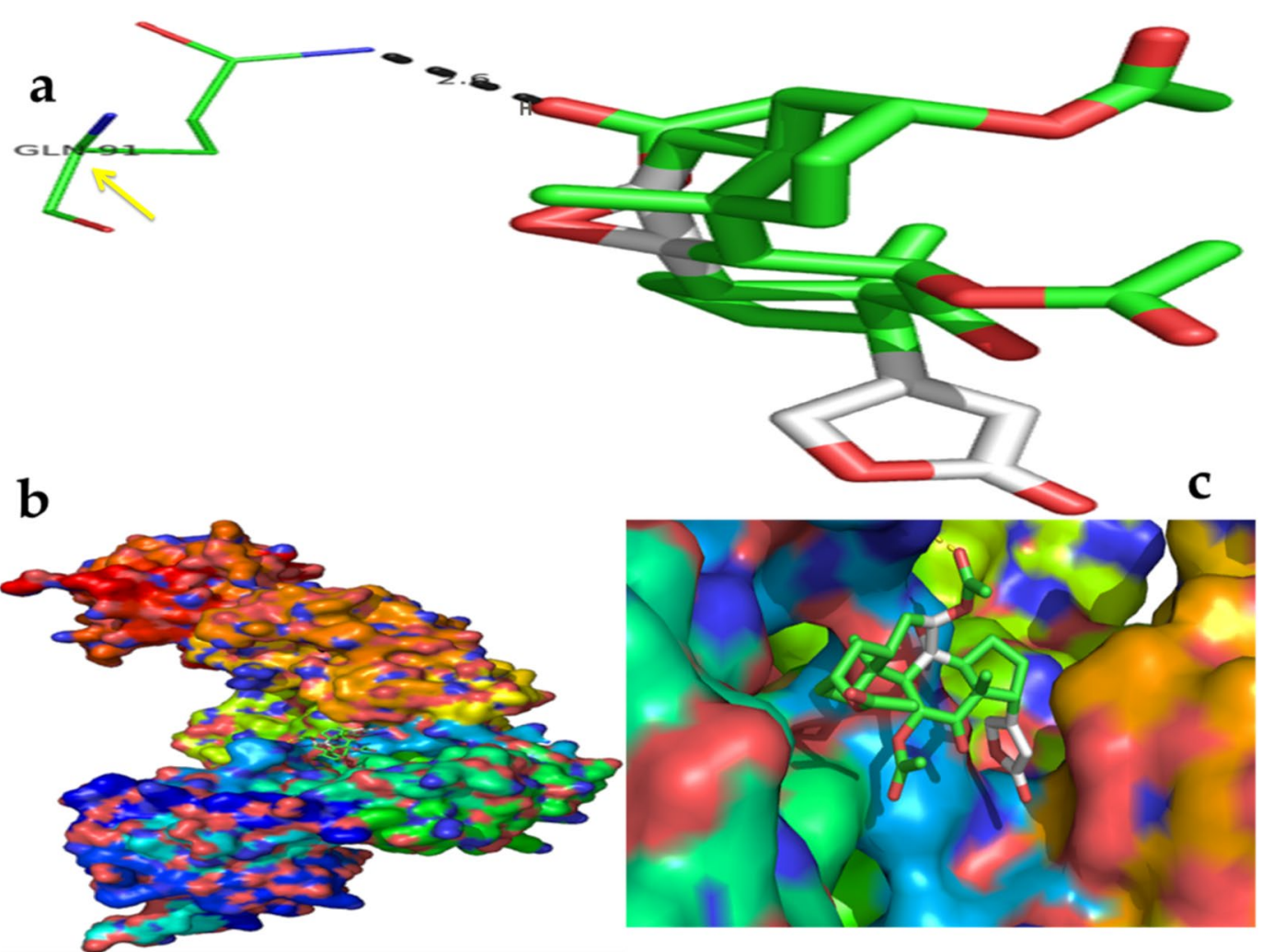

GLN

Figure 11. In silico molecular docking shows the binding interaction of serverogenin acetate ([3,12-diacetyloxy-10,14-dimethyl-13-oxo-15-(5-oxo-2H-furan-3-yl)-2-oxapentacyclo[9.7.0.01,3.05,10.014,18] octadecan-7-yl] acetate) compound with viral target protein (1rev) based on the binding energy generated by AutoDock program. (a) A hydrogen-bond interaction is formed by GLN91 (orange arrow) in the serverogenin acetate compound. (b) A close-up view of serverogenin acetate compound that binds to the viral target protein. (c) Binding pose of viral target protein with serverogenin acetate compound.

Gas chromatography-mass spectrometry (GC-MS) analysis. GC-MS analyses of leaf and rhizome extracts were carried out using the Perkin-Elmer Clarus 680 system (Perkin-Elmer, Inc. U.S.A) equipped with a fused silica column, packed with Elite-5MS) capillary column ( $30 \mathrm{~m}$ in length $\times 250 \mu \mathrm{m}$ in diameter $\times 0.25 \mu \mathrm{m}$ in thickness). Pure helium gas (99.99\%) was used as the carrier gas at a constant flow rate of $1 \mathrm{~mL} / \mathrm{min}$. For GCMS spectral detection, an electron ionization energy method was adopted with high ionization energy of $70 \mathrm{eV}$ (electron Volts) with $0.2 \mathrm{~s}$ of scan time and fragments ranging from 40 to $600 \mathrm{~m} / \mathrm{z}$. The injection quantity of $1 \mu \mathrm{L}$ was used (split ratio 10:1), and the injector temperature was maintained at $250^{\circ} \mathrm{C}$ (constant). The column oven temperature was set at $50^{\circ} \mathrm{C}$ for $3 \mathrm{~min}$, raised at $10^{\circ} \mathrm{C}$ per min up to $280^{\circ} \mathrm{C}$, and final temperature was increased to $300{ }^{\circ} \mathrm{C}$ for $10 \mathrm{~min}$. The contents of phytochemicals present in the test samples were identified based on comparison of their retention time (min), peak area, peak height and mass spectral patterns with those spectral database of authentic compounds stored in the National Institute of Standards and Technology (NIST) library ${ }^{60}$.

Molecular docking. All the compounds identified by GC-MS analysis were analyzed to study their putative antibacterial, antifungal, antiviral, antioxidant and antidiabetic activities through docking studies. The structures of the compounds were obtained from Pubchem database (https://pubchem.ncbi.nlm.nih.gov/) as 


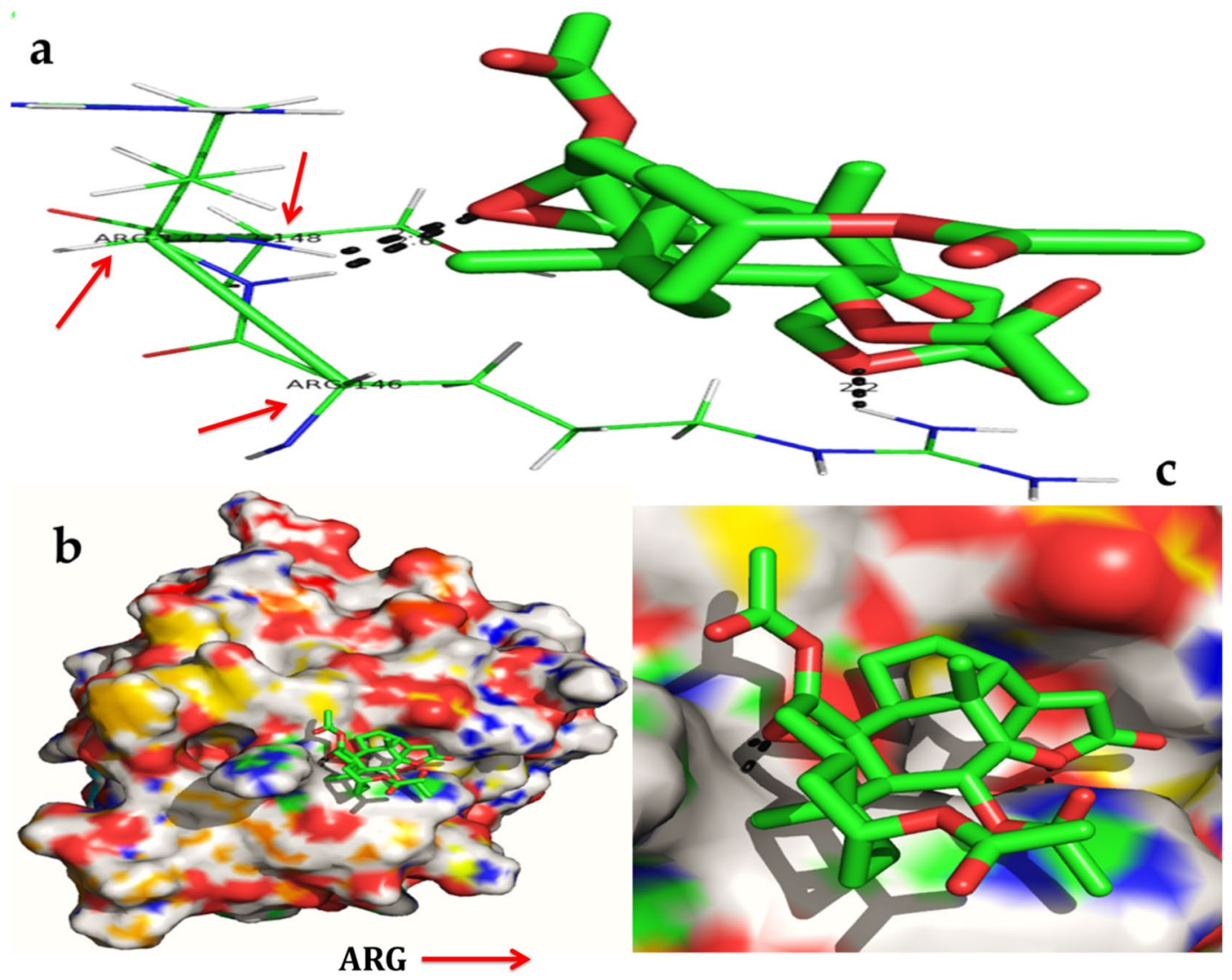

Figure 12. In silico molecular docking shows the binding interaction of serverogenin acetate ([3,12-diacetyloxy-10,14-dimethyl-13-oxo-15-(5-oxo-2H-furan-3-yl)-2-oxapentacyclo[9.7.0.01,3.05,10.014,18] octadecan-7-yl] acetate) compound with antioxidant target protein (2he3) based on the binding energy generated by AutoDock program. (a) A hydrogen-bond interaction is formed by O in ARG146, ARG147 and ARG148 (red arrow) in the serverogenin acetate compound. (b) A close-up view of serverogenin acetate compound that binds to the anti-oxidant target protein. (c) Binding pose of antioxidant target protein with serverogenin acetate compound.

sdf file and converted into Mol, PDBQT and PDB file formats using OPEN BABEL software (https://openbabel. org/wiki/Main_Page). The three-dimensional (3D) structures of phytocompounds were optimized for docking conformation study. The structure-based molecular docking was performed to study the protein ligand interactions of the phytocompounds with bacterial (PDB code:5iwm), fungal (PDB code:4i9p), viral (PDB code:1 rev), antioxidant (PDB code:2he3) and diabetic [ $\alpha$-glucosidase (PDB code:5nn3), $\alpha$-amylase (PDB code:i-tasser) and aldose reductase (PDB code:4ys1)] target proteins obtained from the Research Collaboratory for Structural Bioinformatics (RCSB) Protein Data Bank (https://www.rscb.org) ${ }^{61}$ using the iGEMDOCK 2.1 software (https:// gemdock.life.nctu.edu.tw/dock/igemdock.php ${ }^{62}$ and AutoDock program ${ }^{63}$.

Initially, all the target proteins and compounds were prepared by assigning hydrogen bonds, bond orders, charges and flexible torsions. Screening of these compounds for protein ligand interactions was carried out using the iGEMDOCK program with the preset parameters: population size of 150 was set with 60 generations and two solutions were selected for customized docking parameter. From the customized docking study, the probable binding conformations of phytocompounds were determined based on the iGEMDOCK total energy.

The best binding conformation of phytocompounds against target proteins were determined based on the lowest total binding energy among the different conformations generated. The identified phytocompounds were imported into the iGEMDOCK graphical user interface and were sorted by the post-docking analysis based on their binding energies and compound fitness score measured by the iGEMDOCK docking algorithm ${ }^{64}$. To determine the relative strengths of the binding interactions of the best identified phytocompound, screening for its best binding pose and the series of energy values, such as binding energy, ligand efficiency, inhibition constant and Van der Waals (VDW) + hydrogen bonding (Hbond) + desolvation energy of each target protein, were analyzed using the AutoDOCK program. Furthermore, the detailed interactions between the best phytocompound identified, and its binding sites with bacterial, fungal, viral and diabetic target proteins were visualized in PyMol 3D visualization ${ }^{65}$. 


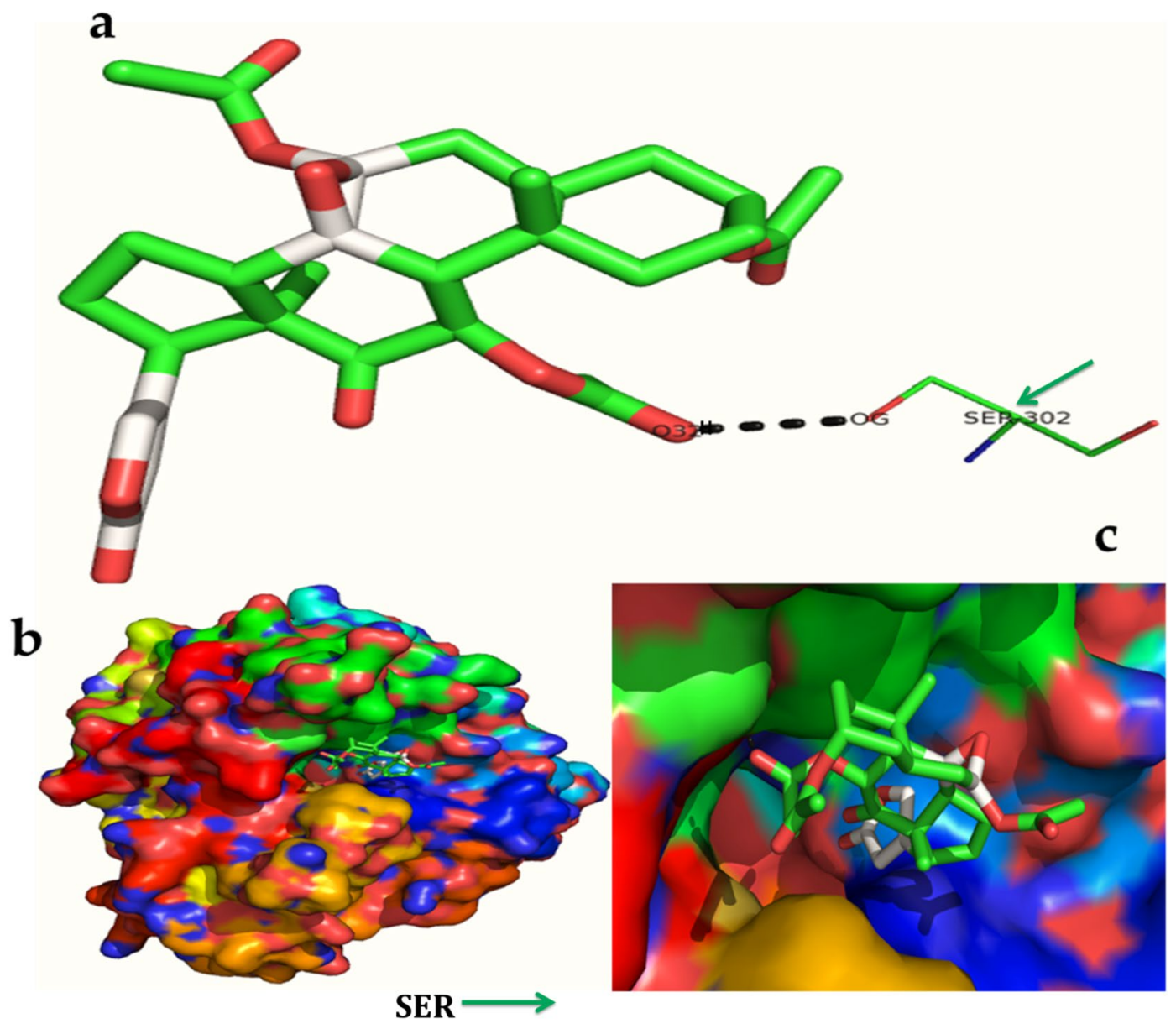

Figure 13. In silico molecular docking shows the binding interaction of serverogenin acetate ([3,12-diacetyloxy-10,14-dimethyl-13-oxo-15-(5-oxo-2H-furan-3-yl)-2-oxapentacyclo[9.7.0.01,3.05,10.014,18] octadecan-7-yl] acetate) compound with aldose reductase target protein (4ysl) based on the binding energy generated by AutoDock program. (a) A hydrogen-bond interaction is formed by O3 and OG in SER302 (green arrow) in the serverogenin acetate compound. (b) A close-up view of serverogenin acetate compound that binds to the aldose reductase target protein. (c) Binding pose of aldose reductase target protein with serverogenin acetate compound.

Antimicrobial activity. Antimicrobial activity of leaf and rhizome extracts was determined using the agar well diffusion method. The antibacterial activity of leaf and rhizome extracts of A. nilgiricum was carried out against Ralstonia solanacearum (RS5-KF924743), Staphylococcus aureus (NCIM 2079), Pseudomonas aeruginosa (NCIM 2200), Escherichia coli (NCIM 2256), Klebsiella pneumonia (NCIM 2957), Bacillus subtilis (NCIM 2724), Salmonella typhimurium (NCIM 2501), and Proteus vulgaris (NCIM 2027) which were procured (except $R$. solanacearum) from National Collection of Industrial Microorganism (NCIM), NCL, Pune, India. Streptomycin $(30 \mu \mathrm{g} / \mathrm{mL})$ was used as a positive control. The antifungal activity of leaf and rhizome extracts of A. nilgiricum was carried out against Aspergillus flavus (NCIM 1028), Aspergillus terreus (NCIM 1325), Aspergillus niger (NCIM 1196), F. oxysporum (NCIM 1281), Alternaria alternata (NCIM 718) and Pyricularia oryzae were obtained from the NCIM, NCL, Pune India. Nystatin was used as a positive control.

Antioxidant activity. The 2,2-diphenyl-1-picryl-hydrazyl-hydrate (DPPH) is a generally used method to evaluate the free radical scavenging ability of natural compounds. The free radical scavenging activity of the leaf and rhizome extracts of $A$. nilgiricum were measured against $\mathrm{DPPH}^{66}$. The Ferric reducing antioxidant power assay (FRAP) was used to measure the total antioxidant power from the leaf and rhizome extracts ${ }^{67}$.

Antidiabetic activities. The $\alpha$-amylase inhibition assay from $A$. nilgiricum leaf and rhizome extracts was determined using the 3,5-dinitrosalicylic acid method ${ }^{68}$. The a-glucosidase inhibition activity of A. nilgiricum leaf and rhizome extracts was measured using the method of Eom et al. ${ }^{69}$. Acarbose was used as positive control. The aldose reductase (AR) inhibition assay from A. nilgiricum leaf and rhizome extracts was determined according to Suryanarayana et al. ${ }^{70}$ using Quercetin as positive control. The inhibitory action was stated as the half maximal inhibitory concentration $\left(\mathrm{IC}_{50}\right)$, which is a measure of the effectiveness of the extract in inhibiting enzymes activities. 

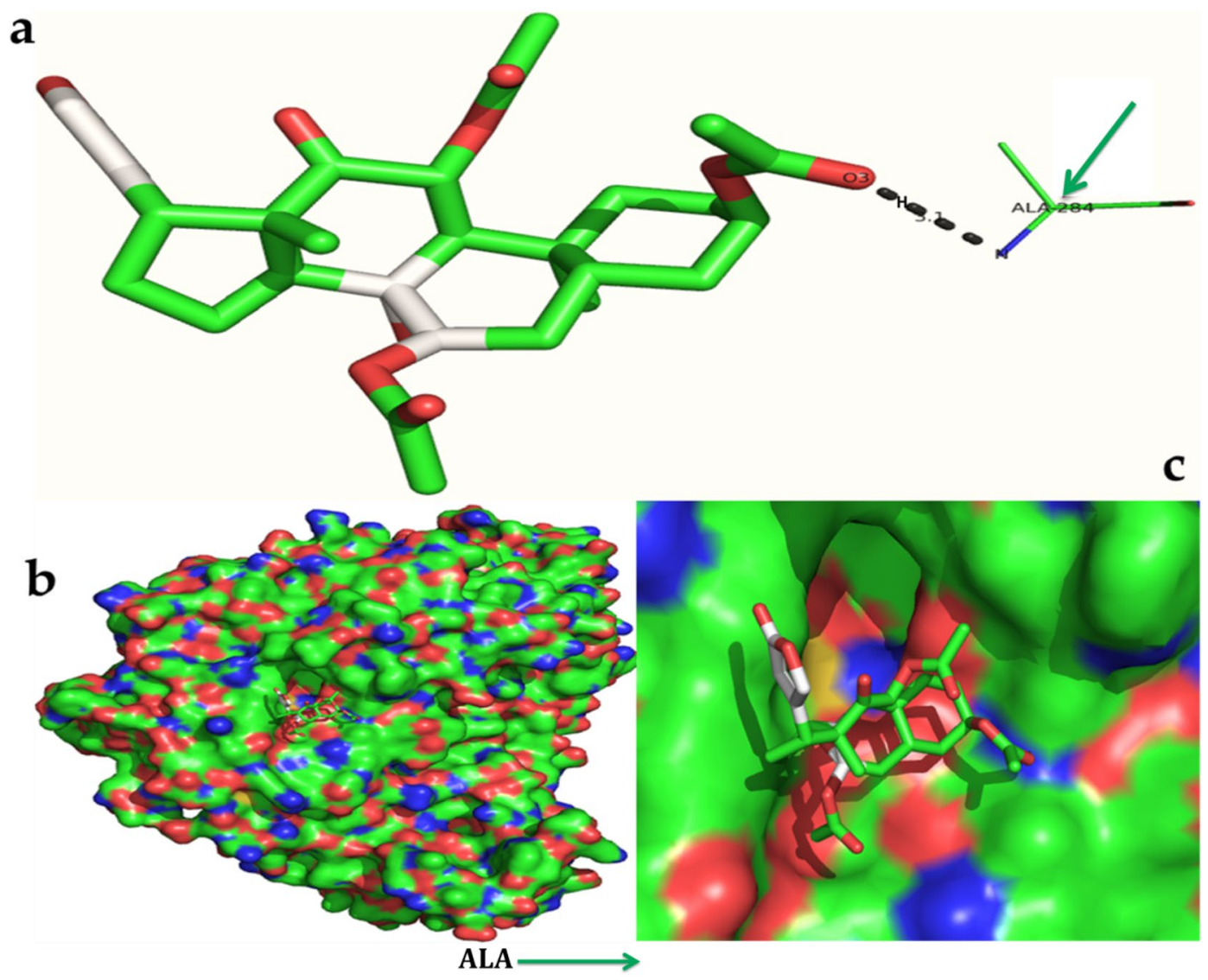

Figure 14. In silico molecular docking shows the binding interaction of serverogenin acetate ([3,12-diacetyloxy-10,14-dimethyl-13-oxo-15-(5-oxo-2H-furan-3-yl)-2-oxapentacyclo[9.7.0.01,3.05,10.014,18] octadecan-7-yl] acetate) compound with a-glucosidase target protein (5nn3) based on the binding energy generated by AutoDock program. (a) A hydrogen-bond interaction is formed by O3 in ALA284 (bright green arrow) in the serverogenin acetate compound. (b) A close-up view of serverogenin acetate compound that binds to the $\alpha$-glucosidase target protein. (c) Binding pose of $\alpha$-glucosidase target protein with serverogenin acetate compound. 


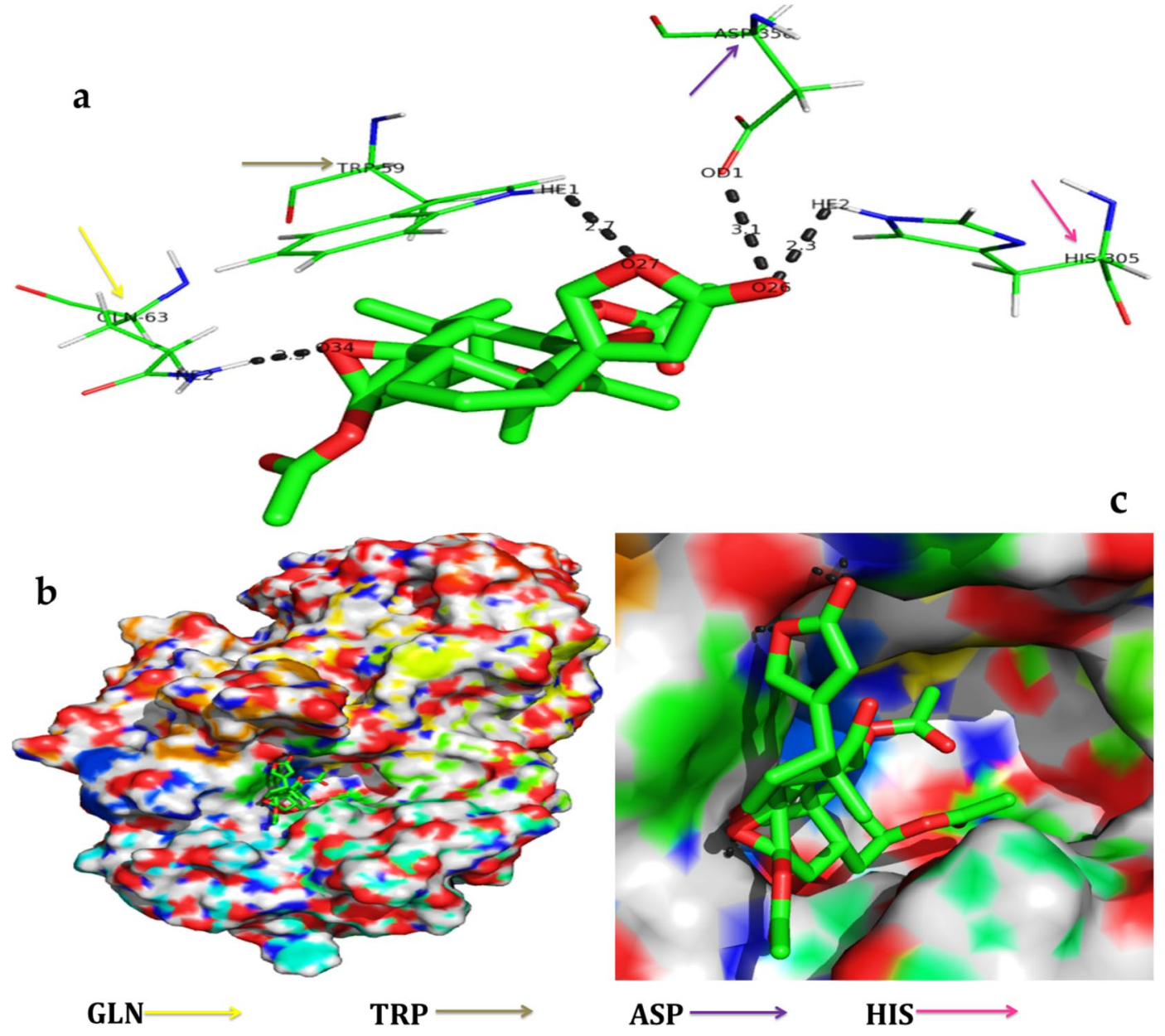

Figure 15. In silico molecular docking shows the binding interaction of serverogenin acetate ([3,12-diacetyloxy-10,14-dimethyl-13-oxo-15-(5-oxo-2H-furan-3-yl)-2-oxapentacyclo[9.7.0.01,3.05,10.014,18] octadecan-7-yl] acetate) compound with $\alpha$-amylase target protein (i-tasser) based on the binding energy generated by AutoDock program. (a) A hydrogen-bond interaction is formed by O26 in HE2, O27 in HE1, O34 in HE2, TRP59 (grey arrow), GLN63 (orange arrow), HIS305 (pink arrow) and ASP356 (purple arrow) in the serverogenin acetate compound. (b) A close-up view of serverogenin acetate compound that binds to the $\alpha$-amylase target protein. (c) Binding pose of $\alpha$-amylase target protein with serverogenin acetate compound.

Received: 18 June 2020; Accepted: 17 September 2020

Published online: 02 October 2020

\section{References}

1. Philomena, G. Concerns regarding the safety and toxicity of medicinal plants-an overview. J. Appl. Pharmaceut. Sci. 1, 40-44 (2011).

2. Sahoo, N. \& Manchikant, P. Herbal drug regulation and commercialization: an Indian industry perspective. J. Altern. Complement. Med. 19, 957-963 (2013).

3. Yuan, H., Ma, Q., Ye, L. \& Piao, G. The traditional medicine and modern medicine from natural products. Molecules 21, 559 (2016).

4. Anand, U., Jacobo-Herrera, N., Altemimi, A. \& Lakhssassi, N. A comprehensive review on medicinal plants as antimicrobial therapeutics: potential avenues of biocompatible drug discovery. Metabolites 9, 258 (2019).

5. Semwal, D. K. et al. Status of Indian medicinal plants in the International Union for Conservation of Nature and the future of Ayurvedic drugs: Shouldn't think about Ayurvedic fundamentals?. J. Integr. Med. 17, 238-243 (2019).

6. Pandey, M. M., Rastogi, S. \& Rawat, A. K. Indian traditional ayurvedic system of medicine and nutritional supplementation. Evid. Based Complem. Altern. Med. https://doi.org/10.1155/2013/376327 (2013).

7. Pandey, M. M., Rastogi, S. \& Rawat, A. K. Indian herbal drug for general healthcare: an overview. Internet. J. Altern. Med. 6, 1-4 (2008).

8. Sreenivasa, N. et al. Biosynthesis and characterization of Dillenia indica-mediated silver nanoparticles and their biological activity. App. Organomet. Chem. 34, e5567 (2020).

9. Malongane, F., McGaw, L. J. \& Mudau, F. N. The synergistic potential of various teas, herbs and therapeutic drugs in health improvement: a review. J. Sci. Food Agri. 97, 4679-4689 (2017).

10. Keskes, H. et al. LC-MS-MS and GC-MS analyses of biologically active extracts and fractions from Tunisian Juniperus phoenice leaves. Pharm. Biol. 55, 88-95 (2017).

11. Yadav, R., Khare, R. K. \& Singhal, A. Qualitative phytochemical screening of some selected medicinal plants of Shivpuri District (MP). Int. J. Life Sci. Sci. Res. 3, 844-847 (2017). 
12. Juszczak, A. M., Zovko-Končić, M. \& Tomczyk, M. Recent trends in the application of chromatographic techniques in the analysis of Luteolin and its derivatives. Biomolecules 9, 731 (2019).

13. Satapute, P., Murali, K. P., Kurjogi, M. \& Jogaiah, S. Physiological adaptation and spectral annotation of Arsenic and Cadmium heavy metal-resistant and susceptible strain Pseudomonas taiwanensis. Environ. Pollut. 251, 555-563 (2019).

14. Fan, S., Chang, J., Zong, Y., Hu, G. \& Jia, J. GC-MS analysis of the composition of the essential oil from Dendranthema indicum Var. Aromaticum using three extraction methods and two columns. Molecules 23, 576 (2018).

15. Razack, S., Kumar, K. H., Nallamuthu, I., Naika, M. \& Khanum, F. Antioxidant, biomolecule oxidation protective activities of Nardostachys jatamansi DC and its phytochemical analysis by RP-HPLC and GC-MS. Antioxidants 4, 185-203 (2015).

16. Thomas, V. P., Sabu, M. \& Prabhu Kumar, K. M. Amomum nilgiricum (Zingiberaceae), a new species from Western Ghats India. Phyto Keys 8, 99-104 (2012).

17. Kumar, K. M. P., Asish, G. R., Sabu, M. \& Balachandran, I. Significance of gingers (Zingiberaceae) in Indian System of MedicineAyurveda: an overview. Anc. Sci. Life. 32, 253-261 (2013).

18. Kress, W. J., Prince, L. M. \& Williams, K. J. The phylogeny and new classification of the gingers (Zingiberaceae): Evidence from molecular data. Am. J. Bot. 89, 1682-1696 (2002).

19. Fang, J., Liu, C., Wang, Q., Lin, P. \& Cheng, F. In silico polypharmacology of natural products. Brief. Bioinform. 19, 1153-1171 (2018).

20. Sliwoski, G., Kothiwale, S., Meiler, J. \& Lowe, E. W. Computational methods in drug discovery. Pharmacol. Rev. 66, 334-395 (2014).

21. Loza-Mejía, M. A., Salazar, J. R. \& Sánchez-Tejeda, J. F. In Silico studies on compounds derived from Calceolaria: phenylethanoid glycosides as potential multitarget inhibitors for the development of pesticides. Biomolecules 8, 121 (2018).

22. Lee, K. \& Kim, D. In-Silico molecular binding prediction for human drug targets using deep neural multi-task learning. Genes 10, $906(2019)$

23. Bharathi, A. et al. In silico molecular docking and in vitro antidiabetic studies of dihydropyrimido (4,5-a] acridin-2-amines). Bio Med. Res. Int. 1, 1-10 (2014).

24. Fikriya, Z.A., Irawan, M.I. \& Nurhayati, A.P.D. Molecular docking of alkaloid compound sa2014 towards cyclin d1 protein in cancer using firefly algorithm. In J. Physics: Conference Series, IOP Publishing 1366 (1), p.012090 (2019).

25. Kabra, A. et al. Phytochemical composition, antioxidant, and antimicrobial attributes of different solvent extracts from Myrica esculenta Buch.-Ham. Ex. D. Don Leaves. Biomolecules 9, 357 (2019).

26. Tenzin, C., Jeyanthi, P., Kumar, A., Sujesh, S. \& Ramalingam, C. Estimation of bioactive compounds from Saccharum munja extract for the evaluation of antioxidants and antibacterial activities. J. Food Process Technol. 8, 671 (2017).

27. Suma, A. et al. GCMS and FTIR analysis on the methanolic extract of red Vitis vinifera seed. World J. Pharm. Sci. 6, 106-113 (2018).

28. Agneeswari, S., Amutha, S. \& Jaya Arputha Jenishini, J. Characterization and antimicrobial activity of Centella Asiatica. Int. J. Eng. Adv. Technol. 9, 125-131 (2019).

29. Bhamadevi, R., Barkath, T. N., Vijayaraghavan, P. \& Rejiniemon, T. S. GC-MS analysis of phytochemical from Psidium guajava Linn leaf extract and their in vitro antimicrobial activities. Int. J. Pharm. Biol. Sci. 8, 583-589 (2018).

30. Yugandhar, P. \& Nataru, S. Spectroscopic and chromatographic exploration of different phytochemical and mineral contents from Syzygium alternifolim (Wt.) Walp. an endemic, endangered medicinal tree taxon. J. Appl. Pharm. Sci. 7, 73-85 (2017).

31. Penduka, D., Buwa, L., Mayekiso, B., Basson, A. K. \& Okoh, A. I. Identification of the Antilisterial constituents in partially purified column chromatography fractions of Garcinia kola seeds and their interactions with standard antibiotics. Evid. Based Complem. Altern. Med. https://doi.org/10.1155/2014/850347 (2014).

32. Ramakrishnan, S. \& Venkataraman, R. Screening of antioxidant activity, total phenolics and gas chromatography-mass spectrophotometer (GC-MS) study of ethanolic extract of Aporosa lindleyana Baill. Afr. J. Biochem. Res. 5, 360-364 (2011).

33. Senthilkumar, S. \& Vijayakumari, K. Comparative studies on phytochemical and GC-MS analysis of Cassia auriculata linn. and Cardiospermum halicacabum linn. leaf extract-traditional valuable plants. Int. J. Pharm. Res. BioSci. 2, 95-104 (2013).

34. Azizan, K. A., Ghani, N. H. A. \& Nawawi, M. F. GC-MS based metabolomics and multivariate statistical analysis of Wedelia trilobata extracts for the identification of potential phytochemical properties. Plant Omics J. 8, 537-543 (2015).

35. Muhammad, A., Muhammad, S. \& Amer, J. Phytochemical spectrum of Essential Oil of Paganum harmala by GC-MS and antimicrobial activity using sequential solvents fractions and essential oil. Asian J. Chem. 26, 574-578 (2014).

36. Divya, F., Renukumari, Rao, D.V. Isolation and characterization of phytosterols from Butea monosperma (Lamarck) Kuntze. Int. J. Recent Sci. Res.9, 23803-23806 (2018).

37. Sermakkani, M. \& Thangapandian, V. GC-MS analysis of Cassia italica leaf methanol extract. Asian. J. Pharm. Clin. Res. 5, 90-94 (2012).

38. Ismaila, O. N. \& Clement, O. B. Effects of aqueous bark extracts of Khaya grandifoliola and Enantia chlorantha on some biochemical parameters in Swiss Mice. Iran. J. Toxicol. 11, 13-21 (2017).

39. Kathirvel, A., Rai, A. K., Maurya, G. S. \& Sujatha, V. Dryopteris cochleata Rhizome: a nutritional source of essential elements, phytochemicals, antioxidants and antimicrobials. Int. J. Pharm. Pharm. Sci. 6, 179-188 (2014).

40. Ruthiran, P., Lokesh, R. \& Chinnadura, I. S. Phytochemical studies and GC-MS analysis of Spermadictyon suaveolens Roxb. Int. J. Pharm. Pharm. Sci. 9, 143-149 (2017).

41. Mohamed, Z. Z. \& Benedict, S. Phytochemical constituents of the leaves of Leucaena leucocephala from Malaysia. Int. J. Pharm. Pharm. Sci. 8, 174-179 (2016).

42. Askal, M. \& Anton Restu, P. Chemical composition of essential oil and hexane extract and antioxidant activity of various extracts of Acmella uliginosa (Sw.) Cass flowers from Indonesia. Agric. Nat. Res. 50, 264-269 (2016).

43. Ewa, W. B. \& Jolanta, D. Essential oils and hydrophilic extracts from the leaves and flowers of Succisa pratensis Moench and their biological activity. R. Pharm. Soc. J. Pharm. Pharmacol. 69, 1531-1539 (2017).

44. Senthilkumar, N., Murugesan, S. \& Vijayalakshmi, K. B. GC-MS analysis of Trichilia connaroides (Wight \& Arn.) Bentv (Meliaceae): a tree of ethnobotanical records. Asian J. Plant Sci. Res. 2, 207-211 (2012).

45. Priyanka, C., Kumar, P., Bankar, S. P. \& Karthik, L. In vitro antibacterial activity and gas chromatography-mass spectroscopy analysis of Acacia karroo and Ziziphus mauritiana extracts. J. Taibah Univ. Sci. 9, 13-19 (2015).

46. Ashish Kumar, G. \& Bhat, J. L. GC-MS analysis of methanol extract of Acacia nilotica (L.) Leaves. Int. J. Pharma. Chem. 6, 50-53 (2016).

47. Jiji, P. G. \& Subin, M. P. Qualitative phytochemical screening and GC-MS analysis in the leaf methanolic extracts of Kamettia caryophyllata (Roxb.) Nicolson and Suresh. Paripex Indian J. Res. 6, 470-479 (2017).

48. Karthikeyan, K. \& Dhanapal, C. K. GC-MS analysis of methanol extract of Alysicarpus monilifer-whole plant. Der. Pharma. Chem. 8, 563-571 (2016).

49. Santhosh Kumar, B., Manickam, D., Sri Kamatchi, P., Shyama, S. \& Subramaniam, S. GC-MS analysis of an Ayurvedic medicine Modified Arjunarishta. Int. J. Curr. Res. Chem. Pharm. Sci. 4, 46-50 (2017).

50. Ololade, Z. S., Ogunmola, O. O., Kuyooro, S. E. \& Abiona, O. O. Stachytarpheta jamaicensis leaf extract: chemical composition, antioxidant, anti-arthritic, antiinflammatory and bactericidal potentials. J. Sci. Inn. Res. 6, 119-125 (2017).

51. Paulpriya, K., Tresina, P. S. \& Mohan, V. R. (2015) Assessment of bioactive constituents by GC-MS of Crotalaria longipes Wight \& Arn.: an endemic plant. Int. J. Pharmacogn. Phytochem. Res. 6, 1043-1048 (2015).

52. Thanga Krishna, K. S., Muthukumarasamy, S. \& Mohan, V. R. GC-MS analyses of ethanol extract of Sarcostemma secamone (L) bennet (Asclepiadaceae). Sci. Res. 2, 187-191 (2012). 
53. Narasimhamurthy, K., Chandra Nayaka, S., Soumya, K., Brijesh, S. \& Niranjana, S. R. Phytochemical screening and antimicrobial activity of leaf extracts of Amomum nilgiricum (Thomas) (Zingiberaceae) from Western Ghats, India. J. Biol. Prod. Nat. 7, 311-330 (2017).

54. Shrestha, R. L. GC-MS analysis, antibacterial and antioxidant study of Amomum subulatum. J. Med. Plants Stud. 5, 126-129 (2017).

55. Sharma, V., Lohia, N., Handa, V. \& Baranwal, N. Amomum subulatum seed extract exhibit antioxidant, cytotoxic and immunesupressive effect. Indian J. Biochem. Biophys. 54, 135-139 (2017).

56. Salehi, B. et al. Antidiabetic potential of medicinal plants and their active components. Biomolecules 9,551 (2019).

57. Hawkins, P.; Skillman, G. Ligand based design workflow https:/images.apple.com/ science /pdf/ ligand based design workflow. pdf. 2011.

58. Balavignesh, V., Srinivasan, E. \& Ramesh Babu, N. G. Molecular docking study ON NS5B polymerase of hepatitis c virus by screening of volatile compounds from Acacia concinna and ADMET prediction. Int. J. Pharm. Life Sci. 4, 2548-2558 (2013).

59. Tiwari, P., Kumar, B., Kaur, M., Kaur, G. \& Kaur, H. Phytochemical screening and extraction: a review. Int. Pharm. Sci. 1, 98-106 (2011).

60. NIST Chemistry Web Book, Mallard, W.G., Linstrom, P.J., Eds. NIST Standard Reference Database, National Institute of Standards and Technology, (https://webbook.nist.gov). (2008).

61. Kurjogi, M. et al. Computational modeling of the Staphylococcal enterotoxins and their interaction with natural antitoxin compounds. Int. J. Mol. Sci. 19, 133 (2018).

62. Neema, T., Kumud, P. \& Bhasker, P. Docking studies of grayanotoxin as potential inhibitor for major virulent proteins of encephalitis virus. Int. J. Pharmtechres. 7, 156-164 (2015).

63. Morris, G. M. et al. AutoDock4 and AutoDockTools4: automated docking with selective receptor flexibility. J. Comput. Chem. 30, 2785-2791 (2009).

64. Iheagwam, F. N., Ogunlana, O. O., Ogunlana, O. E., Isewon, I. \& Oyelade, J. Potential anti-cancer flavonoids isolated from Caesalpinia bonduc young twigs and leaves: Molecular docking and in silico studies. Bioinform. Biol. Insights. 13, 1-16 (2019).

65. Lill, M. A. \& Danielson, M. L. Computer-aided drug design platform using PyMoL. J. Comput. Aided Mol. Disc. 25, 13-19 (2010).

66. Brand-Williams, W., Cuvelier, M. E. \& Berset, C. Use of a free radical method to evaluate antioxidant activity. Food Sci. Technol. 28, 25-30 (1995).

67. Zhao, H. et al. Evaluation of antioxidant activities and total phenolic contents of typical malting barley varieties. Food Chem. 107, 296-304 (2008).

68. Miller, G. L. Use of dinitrosalicylic acid reagent for determination of reducing sugar. Anal. Chem. 31, 426-428 (1959).

69. Eom, S. H. et al. $\alpha$-Glucosidase-and $\alpha$-amylase-inhibitory activities of phlorotannins from Eisenia bicyclis. J. Sci. Food Agric. 92, 2084-2090 (2012).

70. Suryanarayana, P., Kumar, P. A., Saraswat, M., Petrash, J. M. \& Reddy, G. B. Inhibition of aldose reductase by tannoid principles of Emblica officinalis: implications for the prevention of sugar cataract. Mol. Vis. 10, 148-154 (2004).

\title{
Acknowledgments
}

This work was supported by University Grant Commission (UGC), New Delhi, Post-Doctoral Fellowship (No. F/PDFSS-2014-15-ST-KAR-7487). The authors also thankful to Department of Forests and Wildlife, Govt. of Kerala for providing necessary forest permission. We are also thankful to M. Sabu, Professor, Department of Botany, University of Calicut for the help regarding specimen information and collection. We are also thankful to the laboratory facilities provided at Department of studies in Biotechnology, University of Mysore, Mysore, and also to the laboratory of Plant Healthcare and Diagnostics, PG Department of Biotechnology and Microbiology, Karnatak University, Dharwad, India.

\section{Authors Contributions}

S.J. were involved in conceptualization, supervision project administration and funding acquisition. N.K. and A.C.U were responsible for methodology, investigation, resources, data curation and writing the original draft manuscript. N.K., S.K. and C.K.P. were involved in data curation and visualization. A.C.U., S.C. and S.J. were responsible for software, validation, formal analysis, writing, editing and reviewing the manuscript.

\section{Competing interests}

The authors declare no competing interests.

\section{Additional information}

Correspondence and requests for materials should be addressed to S.J.

Reprints and permissions information is available at www.nature.com/reprints.

Publisher's note Springer Nature remains neutral with regard to jurisdictional claims in published maps and institutional affiliations.

\begin{abstract}
Open Access This article is licensed under a Creative Commons Attribution 4.0 International License, which permits use, sharing, adaptation, distribution and reproduction in any medium or format, as long as you give appropriate credit to the original author(s) and the source, provide a link to the Creative Commons licence, and indicate if changes were made. The images or other third party material in this article are included in the article's Creative Commons licence, unless indicated otherwise in a credit line to the material. If material is not included in the article's Creative Commons licence and your intended use is not permitted by statutory regulation or exceeds the permitted use, you will need to obtain permission directly from the copyright holder. To view a copy of this licence, visit http://creativecommons.org/licenses/by/4.0/.
\end{abstract}

(c) The Author(s) 2020 